Article

\title{
Nanolipid-Trehalose Conjugates and Nano-Assemblies as Putative Autophagy Inducers
}

\author{
Eleonora Colombo ${ }^{1}$, Michele Biocotino ${ }^{1}$, Giulia Frapporti ${ }^{2}$, Pietro Randazzo ${ }^{3}$, \\ Michael S. Christodoulou ${ }^{4}$, Giovanni Piccoli ${ }^{2}$, Laura Polito ${ }^{5}\left({ }^{\circ}\right.$, Pierfausto Seneci ${ }^{1, *}$ \\ and Daniele Passarella ${ }^{1, *}$ (i) \\ 1 Dipartimento di Chimica, Università degli Studi di Milano, Via Golgi 19, 20133 Milano, Italy \\ 2 CIBIO, Università di Trento, Via Sommarive 9, 38123 Povo (TN), Italy \\ Promidis Srl, San Raffaele Scientific Research Park, Torre San Michele 1, Via Olgettina 60, 20132 Milan, Italy \\ 4 DISFARM, Sezione di Chimica Generale e Organica "A. Marchesini", Universitdegli Studi di Milano, \\ via Venezian 21, 20133 Milano, Italy \\ 5 ISTM-CNR, via Fantoli 16/15, 20138 Milan, Italy \\ * Correspondence: pierfausto.seneci@unimi.it (P.S.); daniele.passarella@unimi.it (D.P.); \\ Tel.: +39-02-5031-4060 (P.S.); +39-02-5031-4081 (D.P.)
}

Received: 12 June 2019; Accepted: 8 August 2019; Published: 20 August 2019

\begin{abstract}
The disaccharide trehalose is an autophagy inducer, but its pharmacological application is severely limited by its poor pharmacokinetics properties. Thus, trehalose was coupled via suitable spacers with squalene (in 1:2 and 1:1 stoichiometry) and with betulinic acid (1:2 stoichiometry), in order to yield the corresponding nanolipid-trehalose conjugates 1-Sq-mono, 2-Sq-bis and 3-Be-mono. The conjugates were assembled to produce the corresponding nano-assemblies (NAs) Sq-NA1, Sq-NA2 and Be-NA3. The synthetic and assembly protocols are described in detail. The resulting NAs were characterized in terms of loading and structure, and tested in vitro for their capability to induce autophagy. Our results are presented and thoroughly commented upon.
\end{abstract}

Keywords: nano-assemblies; trehalose; squalene; betulinic acid; autophagy induction

\section{Introduction}

Nano-vectors are used as therapeutics and diagnostics [1,2]. Iron-based [3], gold-based [4] and silica-based inorganic nano-vectors [5] were tested as being a diagnostic (magnetic resonance imaging reagents/MRIs [6]), or as therapeutics (hyperthermia against tumors [7], iron replacement therapies [8]). Liposome- [9], micelle- [10] and polymer-based nano-assemblies (NAs) [11] are marketed, mostly as anti-cancer agents [12]. Exploratory efforts [13] up to clinical trials [14] against diseases of the central nervous system (CNS) were reported.

Trehalose $[15,16]$ is a non-reducing disaccharide made by a 1,1 linkage between two D-glucose molecules. It is bio-synthesized in lower organisms [16] to stabilize life processes and support survival in extreme conditions (freezing [17], heat and desiccation [18]). Trehalose induces autophagy in vitro and in vivo [19] and reduces protein misfolding and aggregation in vitro [20] by acting as a chemical chaperone and solvating them [20]. Reduction of aggregated huntingtin (Huntington Disease) [21], synuclein (Parkinson Disease) [20] and amyloid species (Alzheimer Disease) [22] was observed in vitro. Trehalose was tested as a safe, cheap, neuroprotective agent in preclinical and clinical studies [16]. Unfortunately, high $\mathrm{mM}$ trehalose concentrations are needed in vivo for efficacy, due to its high hydrophilicity and due to trehalase enzymes [23], that hydrolyze trehalose in the brush border cells of the small intestine and in the proximal tubules of the kidneys, preventing its oral absorption.

Nano lipid-drug conjugates [24], obtained by the covalent coupling of a drug to bio-compatible lipids, improve pharmacokinetics, decrease toxicity and increase the therapeutic index of the associated 
drugs. In particular, squalene-based amphiphilic conjugates have a proven track record for therapeutic applications [25]. They spontaneously assemble in water into nano-assemblies (NAs), encasing the bioactive payload, they do not show the drug on their surface, minimizing any side effect [26], they are internalized by cells via endocytic pathways [27] and release the free drug at its site of action, when a biologically labile linkage is used [28]. We also selected betulinic acid-based conjugates, a less studied but promising class of self-assembling NAs [29,30].

In recent years we worked on anticancer drug-containing self-assembling drug conjugates that spontaneously form NAs in aqueous media [31]. We reported NAs composed by conjugate releasable compounds [32]; by single and dual drug fluorescent hetero-NAs [33,34], by dual drug hetero-NAs (cyclopamine/taxol [34], cyclopamine/doxorubicin [35], ecdysteroid/doxorubicin [36]), and by self-assembling conjugate dual drug NAs [37]. We prepared compounds containing a squalene [31-35] or a 4-(1,2-diphenylbut-1-en-1-yl)aniline tail $[37,38]$ that leads to NAs ability to self-assemble in water. We recently reported the assembly and characterization of squalene-thiocolchicine NAs that release cytotoxic, free thiocolchicine in cancer cells through a disulfide bond or a $p$-hydroxybenzyl moiety [38]. Here we describe the synthesis of two squalene-trehalose conjugates 1-Sq-mono and 2-Sq-bis, and of a betulinic acid-trehalose conjugate 3-Be-mono, their assembly and characterization as NAs (respectively mono-Sq-NA1, bis-Sq-NA2 and mono-Be-NA3, Figure 1), and their effects in biological assays. We measured cell viability to determine the safety of our NAs, while autophagy induction was selected as a validated neuroprotective mechanism of action in multiple neurodegenerative diseases [39]. Trehalose-containing NAs may significantly increase the weak, high $\mathrm{mM}$ effects of trehalose on autophagy induction [19], by facilitating its cellular internalization, and by releasing after NA disassembly/ester hydrolysis.

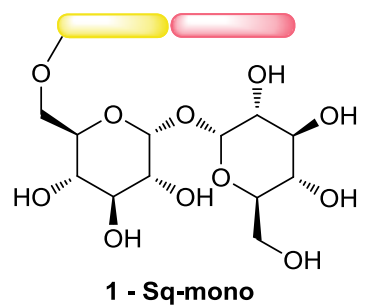

1 - Sq-mono

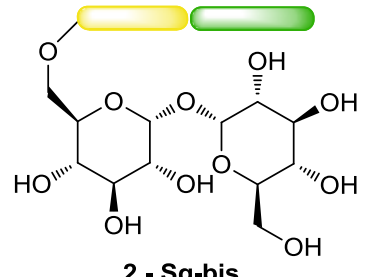

2 - Sq-bis<smiles>C#CCOCC1OC(OC2C(COC)OC(COC)C(O)C2O)C(O)[C@@H](O)[C@@H]1O</smiles>

3 - Be-mono

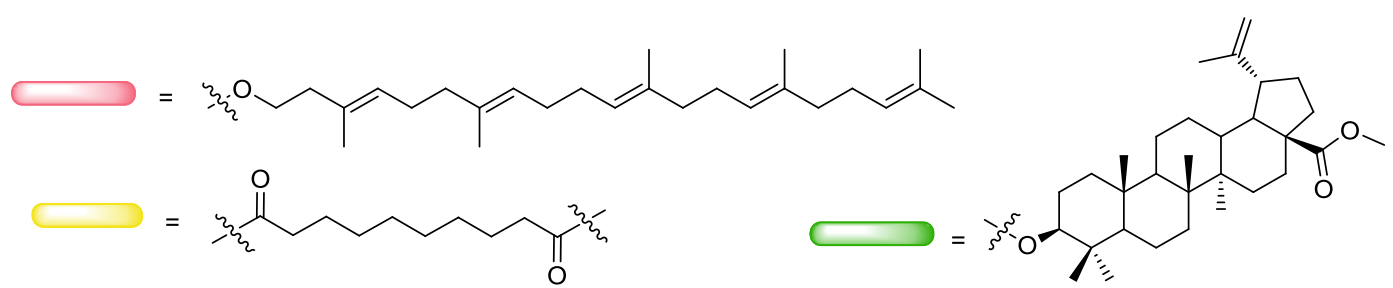

Figure 1. Chemical structure of nanolipid-trehalose conjugates 1-Sq-mono, 2-Sq-bis, and 3-Be-mono.

\section{Materials and Methods}

\subsection{Synthesis-General}

Each reaction was carried out in oven-dried glassware, using dry solvents under a nitrogen atmosphere. Unless otherwise stated, these solvents were purchased from Sigma Aldrich Italy (Milan, Italy) and used without further purification. Chemical reagents were purchased from Sigma Aldrich, and used as such. Thin layer chromatography (TLC) was performed on Merck-pre-coated 60F 254 plates. Reactions were monitored by TLC on silica gel, with detection by UV light ( $254 \mathrm{~nm}$ ), or by charring either with a $1 \%$ permanganate solution or a $50 \% \mathrm{H}_{2} \mathrm{SO}_{4}$ solution. Flash chromatography columns were run using silica gel (240-400 mesh, Merck Italy, Milan, Italy).

${ }^{1} \mathrm{H}-\mathrm{NMR}$ spectra were recorded on Bruker DRX-400 and Bruker DRX-300 instruments (Billerica, MA, USA) in either $\mathrm{CDCl}_{3}, \mathrm{CD}_{3} \mathrm{OD}$ or DMSO-d6. ${ }^{13} \mathrm{C}-\mathrm{NMR}$ spectra were recorded on the same 
instrumentation (100 and $75 \mathrm{MHz}$ ) in either $\mathrm{CDCl}_{3}, \mathrm{CD}_{3} \mathrm{OD}$ or DMSO-d6. Chemical shifts $(\delta)$ for proton and carbon signals are quoted in parts per million (ppm) relative to tetramethylsilane (TMS), which was used as an internal standard. Electrospray ionization (ESI) MS spectra were recorded with a Waters Micromass Q-Tof micro mass spectrometer (Milford, MA, USA); HR-ESI mass spectra were recorded on a FT-ICR APEX ${ }_{\text {II }}$ (Bruker, Billerica, MA, USA), while EI mass spectra were recorded at an ionizing voltage of $6 \mathrm{kEv}$ on a VG 70-70 EQ. Specific rotations were measured with a P-1030-Jasco polarimeter with $10 \mathrm{~cm}$ optical path cells and $1 \mathrm{~mL}$ capacity $(\mathrm{Na}$ lamp, $\lambda=589 \mathrm{~nm}$ ).

\subsection{Synthesis-Squalene-Trehalose Conjugates 1-Sq-Mono and 2-Sq-Bis}

1-[(2R,3R,4S,5R,6R)-6-\{[(2R,3R,4S,5R,6R)-6-(hydroxymethyl)-3,4,5-tris[(trimethylsilyl)oxy]oxan-2yl]oxy\}-3,4,5-tris[(trimethylsilyl)oxy]oxan-2-yl]methyl10-(3E,7E,11E,15E)-3,7,12,16,20pentamethylhenicosa-3,7,11,15,19-pentaen-1-yl decanedioate/15-mono and 1-[(2R,3R,4S,5R,6R)-6- $\{[(2 R, 3 R, 4 S, 5 R, 6 R)-6-$ $\{[(10-$ oxo-10-\{[(3E,7E,11E,15E)-3,7,12,16,20-penta-methylhenicosa-3,7,11,15,19-pentaen-1-yl]oxy\}decanoyl)oxy]methyl\}-3,4,5-tris[(trimethylsilyl)oxy]oxan-2-yl]oxy\}-3,4,5-tris[(trimethylsilyl)oxy]-oxan2-yl]methyl10-(3E,7E,11E,15E)-3,7,12,16,20-penta-methylhenicosa-3,7,11,15,19-pentaen-1-yl decanedioate/16-bis. EDC. $\mathrm{HCl}(223 \mathrm{mg}, 1.161 \mathrm{mmol})$ and DMAP (5 mg, $0.039 \mathrm{mmol})$ were added under stirring at room temperature (RT) to a solution of hexaTMS-protected trehalose 5 [40] $(300 \mathrm{mg}, 0.387 \mathrm{mmol})$ in anhydrous toluene $(8.3 \mathrm{~mL})$. After $30 \mathrm{~min}$, carboxylated squalene-linker adduct 6 [32] $(221 \mathrm{mg}, 0.387 \mathrm{mmol})$ was added, and the reaction mixture was stirred at $50{ }^{\circ} \mathrm{C}$ overnight. Reaction monitoring (TLC, eluant: 9:1 n-hexane/AcOEt) confirmed the disappearance of hexaTMS-protected trehalose 5. The solvent was then removed under reduced pressure, and the crude oil was purified by flash chromatography (silicagel, eluant: 9:1 n-hexane/AcOEt) to obtain pure 15-mono (232.9 mg, $0.175 \mathrm{mmol}, 45 \%$ yield) and pure 16-bis (101.2 mg, $0.054 \mathrm{mmol}, 14 \%$ yield).

Analytical characterization. 15-mono: ${ }^{1} \mathrm{H}-\mathrm{NMR}\left(\mathrm{CDCl}_{3}, 400 \mathrm{MHz}\right): \delta(\mathrm{ppm})=5.21-5.05(\mathrm{~m}, 5 \mathrm{H})$, $4.94(\mathrm{t}, J=2.8 \mathrm{~Hz}, 2 \mathrm{H}), 4.32(\mathrm{dd}, J=11.8,2.1 \mathrm{~Hz}, 1 \mathrm{H}), 4.12-3.98(\mathrm{~m}, 4 \mathrm{H}), 3.98-3.82(\mathrm{~m}, 3 \mathrm{H}), 3.71(\mathrm{dd}$, $J=6.4,3.4 \mathrm{~Hz}, 2 \mathrm{H}), 3.54-3.41(\mathrm{~m}, 4 \mathrm{H}), 2.41-2.26(\mathrm{~m}, 4 \mathrm{H}), 2.14-1.96(\mathrm{~m}, 16 \mathrm{H}), 1.79-1.67(\mathrm{~m}, 5 \mathrm{H}), 1.62-1.58$ $(\mathrm{m}, 21 \mathrm{H}), 1.37-1.29(\mathrm{~m}, 8 \mathrm{H}), 0.22-0.11(\mathrm{~m}, 54 \mathrm{H}) .{ }^{13} \mathrm{C}-\mathrm{NMR}\left(\mathrm{CDCl}_{3}, 400 \mathrm{MHz}\right): \delta(\mathrm{ppm})=173.33,173.18$, $134.88,134.76,134.07,132.4,132.00,129.28,129.17,124.84,124.57,124.37,93.83,93.67,73.77,73.66$, $73.03,72.05,71.94,70.59,70.10,68.28,63.61,63.24,61.27,39.73(2 C), 35.72,33.98,33.87,29.45,29.04(3 C)$, 28.99, 28.86, 28.18, 26.68, 26.43 (2C), 25.95, 25.01, 24.91, 22.38 (2C), 17.95, 16.28, 16.22, 16.05, (1.35, 1.18, $0.36=18 \mathrm{C})$. HR-ESI-MS: MW 1349.7973 calcd. for $\mathrm{C}_{67} \mathrm{H}_{130} \mathrm{O}_{14} \mathrm{Si}_{6} \mathrm{Na}, \mathrm{MW} 1349.7982$ found. Optical rotation, $[\alpha]_{D}^{20}$ : $-61.9^{\circ}$. 16-bis: ${ }^{1} \mathrm{H}-\mathrm{NMR}\left(\left(\mathrm{CDCl}_{3}, 400 \mathrm{MHz}\right): \delta(\mathrm{ppm})=5.16-5.10(\mathrm{~m}, 10 \mathrm{H}), 4.94(\mathrm{~d}\right.$, $J=3.0 \mathrm{~Hz}, 2 \mathrm{H}), 4.31-4.28(\mathrm{~m}, 2 \mathrm{H}), 4.10-3.99(\mathrm{~d}, J=38.8 \mathrm{~Hz}, 8 \mathrm{H}), 3.95-3.90(\mathrm{~m}, 2 \mathrm{H}), 3.52-3.44(\mathrm{~m}$, $4 \mathrm{H}), 2.38-2.28(\mathrm{~m}, 8 \mathrm{H}), 2.13-1.98(\mathrm{~m}, 36 \mathrm{H}), 1.77-1.68(\mathrm{~m}, 10 \mathrm{H}), 1.66-1.59(\mathrm{~m}, 38 \mathrm{H}), 1.36-1.28(\mathrm{~m}, 16 \mathrm{H})$, $0.21-0.11(\mathrm{~m}, 54 \mathrm{H}) .{ }^{13} \mathrm{C}-\mathrm{NMR}\left(\mathrm{CDCl}_{3}, 400 \mathrm{MHz}\right): \delta(\mathrm{ppm})=173.78(2 \mathrm{C}), 173.62(2 \mathrm{C}), 135.05(2 \mathrm{C}), 134.90$ (2C), 134.82 (2C), 133.65 (2C), 131.15 (2C), $125.07(2 \mathrm{C}), 124.40(4 \mathrm{C}), 124.29(4 \mathrm{C}), 94.40(2 \mathrm{C}), 73.48(2 \mathrm{C})$, $72.67(2 \mathrm{C}), 71.94(2 \mathrm{C}), 70.74(2 \mathrm{C}), 63.94(2 \mathrm{C}), 63.30(2 \mathrm{C}), 39.73(4 \mathrm{C}), 35.80(2 \mathrm{C}), 34.33(2 \mathrm{C}), 34.09$ (2C), $29.68(2 \mathrm{C}), 29.11(6 \mathrm{C}), 28.77$ (2C), 28.25 (4C), $26.91(2 \mathrm{C}), 26.76(2 \mathrm{C}), 26.65(4 \mathrm{C}), 25.67$ (4C), $24.96(2 \mathrm{C})$, $24.74(2 \mathrm{C}), 17.66$ (2C), $16.02(4 \mathrm{C}), 15.85(2 \mathrm{C}),(1.05,0.87,0.44,0.17=18 \mathrm{C})$. HR-ESI-MS: MW 1902.2516 calcd. for $\mathrm{C}_{104} \mathrm{H}_{190} \mathrm{O}_{17} \mathrm{Si}_{6} \mathrm{Na}$, MW 1902.2524 found. Optical rotation, $[\alpha]_{D}^{20}:-43.9^{\circ}$.

1-(3E,7E,11E,15E)-3,7,12,16,20-pentamethylhenicosa-3,7,11,15,19-pentaen-1-yl 10-[(2R,3S,4S, 5R,6R)-3,4,5-trihydroxy-6-\{[(2R,3R,4S,5S,6R)-3,4,5-trihydroxy-6-(hydroxymethyl)oxan-2-yl]oxy\}oxan2-yl]-methyl decanedioate/1-Sq-mono. Acetic acid $(0.1 \mathrm{~mL}, 1.73 \mathrm{mmol})$ was added under stirring at RT to a solution of $15-\mathrm{mono}(230.0 \mathrm{mg}, 0.173 \mathrm{mmol})$ in $\mathrm{MeOH}(3 \mathrm{~mL})$, and the reaction mixture was stirred at $40{ }^{\circ} \mathrm{C}$ overnight. Reaction monitoring (TLC, eluant: 9:1 $n$-hexane/AcOEt) confirmed the disappearance of 15-mono. The solvent was then removed under reduced pressure, and the crude solid was purified by flash chromatography (silicagel, eluant: $85: 15 \mathrm{CH}_{2} \mathrm{Cl}_{2} / \mathrm{MeOH}$ ) to obtain pure target 1-Sq-mono (153.2 mg, $0.171 \mathrm{mmol}$, quantitative yield).

Analytical characterization. ${ }^{1} \mathrm{H}-\mathrm{NMR}(\mathrm{DMSO}-\mathrm{d6}, 400 \mathrm{MHz}): \delta(\mathrm{ppm})=5.00-4.91(\mathrm{~m}, 6 \mathrm{H}), 4.75$ $(\mathrm{d}, J=3.7 \mathrm{~Hz}, 2 \mathrm{H}), 4.72(\mathrm{~d}, J=3.6 \mathrm{~Hz}, 1 \mathrm{H}), 4.64(\mathrm{t}, J=4.7 \mathrm{~Hz}, 2 \mathrm{H}), 4.55(\mathrm{dd}, J=6.2,1.8 \mathrm{~Hz}, 2 \mathrm{H}), 4.22$ 
$(\mathrm{t}, J=6.0 \mathrm{~Hz}, 1 \mathrm{H}), 4.13-4.10(\mathrm{~m}, 1 \mathrm{H}), 3.92(\mathrm{dd}, J=11.8,5.4 \mathrm{~Hz}, 1 \mathrm{H}), 3.83(\mathrm{t}, J=6.6 \mathrm{~Hz}, 2 \mathrm{H}), 3.80-3.76$ (m, 1H), 3.55-3.51 (m, 1H), 3.46-3.40 (m, 3H), 3.38-3.32 (m, 1H), 3.16-3.10 (m, 2H), 3.04-2.98 (m, 2H), 2.1-2.12 (m, 4H), 1.95-1.79 (m, 18H), 1.56-1.49 (m, 5H), 1.44 (bs, 15H), 1.41-1.37 (m, 4H), 1.13 (bs, 8H). ${ }^{13} \mathrm{C}-\mathrm{NMR}$ (DMSO-d6, $100 \mathrm{MHz}$ ): $\delta$ (ppm): $\delta 173.28,173.22,134.83,134.77,134.73,133.97,131.04$, $124.82,124.59,124.52,124.46,124.35,93.81,93.71,73.30,73.26,73.04,72.05,71.94,70.59(2 \mathrm{C}), 70.10,63.63$, $63.56,61.27,40.62,39.64,39.59,39.37,35.71,33.98$ (2C), 31.59, 30.28, 29.46, 29.06, 29.03, 28.91, 28.16, 26.82, 26.67, 26.42 (2C), 25.90, 24.96, 24.89, 17.94, 16.21, 16.06. HR-ESI-MS: MW 917.5602 calcd. for $\mathrm{C}_{49} \mathrm{H}_{82} \mathrm{O}_{14} \mathrm{Na}$, MW 917.5623 found. Optical rotation, $[\alpha]_{D}^{20}:-59.4^{\circ}$.

1-(3E,7E,11E,15E)-3,7,12,16,20-pentamethylhenicosa-3,7,11,15,19-pentaen-1-yl 10-[(2R,3S,4S, 5R,6R)-3,4,5-trihydroxy-6-\{[(2R,3R,4S,5S,6R)-3,4,5-trihydroxy-6-\{[(10-oxo-10-\{[(3E,7E,11E,15E)-3,7,12,16, 20-pentamethylhenicosa-3,7,11,15,19-pentaen-1-yl]oxy\}decanoyl)oxy]methyl\}oxan-2-yl]oxy\}oxan-2-yl]methyl decanedioate/2-Sq-bis. Acetic acid $(30 \mu \mathrm{L}, 0.53 \mathrm{mmol})$ was added under stirring at RT to a solution of 16-bis $(100.0 \mathrm{mg}, 0.053 \mathrm{mmol})$ in $\mathrm{MeOH}(1 \mathrm{~mL})$, and the reaction mixture was stirred at $40{ }^{\circ} \mathrm{C}$ overnight. Reaction monitoring (TLC, eluant: 9:1 $n$-hexane/AcOEt) confirmed the disappearance of 16-bis. The solvent was then removed under reduced pressure, and the crude solid was purified by flash chromatography (silicagel, eluant: $85: 15 \mathrm{CH}_{2} \mathrm{Cl}_{2} / \mathrm{MeOH}$ ) to obtain pure target 2-Sq-bis (69.1 mg, $0.047 \mathrm{mmol}, 90 \%$ yield). Analytical characterization. ${ }^{1} \mathrm{H}-\mathrm{NMR}$ (DMSO-d6, $400 \mathrm{MHz}$ ): $\delta(\mathrm{ppm})=5.12-5.04(\mathrm{~m}, 12 \mathrm{H}), 4.88(\mathrm{~d}, J=4.9 \mathrm{~Hz}, 2 \mathrm{H}), 4.83(\mathrm{~d}, J=3.6 \mathrm{~Hz}, 2 \mathrm{H}), 4.75(\mathrm{~d}, J=6.1 \mathrm{~Hz}, 2 \mathrm{H})$, $4.25-4.21(\mathrm{~m}, 2 \mathrm{H}), 4.03(\mathrm{dd}, J=11.7,5.6 \mathrm{~Hz}, 2 \mathrm{H}), 3.95(\mathrm{t}, J=6.6 \mathrm{~Hz}, 4 \mathrm{H}), 3.92-3.87(\mathrm{~m}, 2 \mathrm{H}), 3.58-3.52$ $(\mathrm{m}, 2 \mathrm{H}), 3.28-3.23(\mathrm{~m}, 2 \mathrm{H}), 3.15-3.09(\mathrm{~m}, 2 \mathrm{H}), 2.28-2.23(\mathrm{~m}, 8 \mathrm{H}), 2.07-1.90(\mathrm{~m}, 36 \mathrm{H}), 1.67-1.60(\mathrm{~m}$, $10 \mathrm{H}), 1.55-1.47$ (m, 38H), 1.24 (s, 16H). ${ }^{13} \mathrm{C}-\mathrm{NMR}$ (DMSO-d6, $\left.100 \mathrm{MHz}\right): \delta(\mathrm{ppm}): 173.90$ (2C), 173.64 (2C), 134.81 (2C), $134.72(2 \mathrm{C}), 134.64$ (2C), 133.63 (2C), 131.08 (2C), $124.94(2 \mathrm{C}), 124.57$ (2C), 124.52 (2C), 124.47 (2C), $124.32(2 \mathrm{C}), 93.75(2 \mathrm{C}), 73.52(2 \mathrm{C}), 72.56(2 \mathrm{C}), 71.93(2 \mathrm{C}), 70.71(2 \mathrm{C}), 63.99(2 \mathrm{C}), 63.34$ (2C), $39.74(4 \mathrm{C}), 39.65(2 \mathrm{C}), 35.86(2 \mathrm{C}), 34.38$ (2C), $34.12(2 \mathrm{C}), 29.74(2 \mathrm{C}), 29.17(2 \mathrm{C}), 29.10(4 \mathrm{C}), 28.28$ (4C), 26.92 (2C), $26.81(2 \mathrm{C}), 26.68(2 \mathrm{C}), 26.65$ (2C), 25.67 (4C), 24.98 (2C), $24.90(2 \mathrm{C}), 17.94(2 \mathrm{C}), 16.24$ (4C), 16.06 (2C). HR-ESI-MS: MW 1470.0147 calcd. for $\mathrm{C}_{86} \mathrm{H}_{142} \mathrm{O}_{17} \mathrm{Na}$, MW 1470.0146 found. Optical rotation, $[\alpha]_{D}^{20}:-33.7^{\circ}$.

\subsection{Synthesis-Betulinic Acid-trehalose Conjugate 3-Be-mono}

Methyl(1R,3aS,5aR,5bR,9S,11aR)-9-hydroxy-5a,5b,8,8,11a-pentamethyl-1-(prop-1-en-2-yl)-icosahydro-1H-cyclopenta[a]chrysene-3a-carboxylate/18. Trimethylsilyl diazomethane ( $2 \mathrm{M}$ in $n$-hexane, $0.66 \mathrm{~mL}, 1.312 \mathrm{mmol}$ ) was added to a solution of betulinic acid $17(500 \mathrm{mg}, 1.093 \mathrm{mmol})$ in dry $\mathrm{MeOH}$ $(10 \mathrm{~mL})$ and dry toluene $(15 \mathrm{~mL})$. The reaction was stirred overnight at RT, and reaction monitoring (TLC, eluant 7:3 $n$-hexane/AcOEt with $1 \% \mathrm{HCOOH}$ ) confirmed the disappearance of starting material 16. The reaction mixture was diluted with diethyl ether $(13 \mathrm{~mL})$ and $10 \% \mathrm{AcOH}(10 \mathrm{~mL})$. The aqueous layer was extracted with diethyl ether $(3 \times 10 \mathrm{~mL})$, and the collected organic phases were washed with sat. $\mathrm{Na}_{2} \mathrm{CO}_{3}(10 \mathrm{~mL})$, dried with $\mathrm{Na}_{2} \mathrm{SO}_{4}$ and then evaporated under reduced pressure to obtain pure 18 as a white solid (486.1 mg, $1.032 \mathrm{mmol}, 95 \%$ yield).

Analytical characterization. ${ }^{1} \mathrm{H}-\mathrm{NMR}\left(\mathrm{CDCl}_{3}, 400 \mathrm{MHz}\right): \delta(\mathrm{ppm})=4.63(\mathrm{bs}, 1 \mathrm{H}), 4.49(\mathrm{bs}, 1 \mathrm{H})$, $3.56(\mathrm{~s}, 3 \mathrm{H}), 3.07(\mathrm{dd}, J=11.2,5.1 \mathrm{~Hz}, 1 \mathrm{H}), 2.89(\mathrm{td}, J=10.9,4.4 \mathrm{~Hz}, 1 \mathrm{H}), 2.20-2.02(\mathrm{~m}, 2 \mathrm{H}), 1.77(\mathrm{dt}$, $J=10.9,5.9 \mathrm{~Hz}, 2 \mathrm{H}), 1.58(\mathrm{~s}, 3 \mathrm{H}), 0.86(\mathrm{~s}, 6 \mathrm{H}), 0.81(\mathrm{~s}, 3 \mathrm{H}), 0.71(\mathrm{~s}, 3 \mathrm{H}), 0.65(\mathrm{~s}, 3 \mathrm{H})$. HR-ESI-MS: MW 493.3658 calcd. for $\mathrm{C}_{31} \mathrm{H}_{50} \mathrm{O}_{3} \mathrm{Na}$, MW 493.3661 found. Optical rotation, $[\alpha]_{D}^{20}:+5.1^{\circ}$.

10-oxo-10-[2-(trimethylsilyl)ethoxy]decanoic acid/19. Trimethylsilylethanol ( $313 \mathrm{~mL}, 2.181 \mathrm{mmol})$, $\mathrm{EDC} . \mathrm{HCl}(559 \mathrm{mg}, 2.909 \mathrm{mmol})$ and DMAP $(89 \mathrm{mg}, 0.727 \mathrm{mmol})$ were added under stirring at RT to a solution of sebacic acid $14(1 \mathrm{~g}, 0.4942 \mathrm{mmol})$ in dry $\mathrm{CH}_{2} \mathrm{Cl}_{2}(25 \mathrm{~mL})$ and pyridine $(2.5 \mathrm{~mL})$. The reaction mixture was stirred at RT overnight. The reaction mixture was then washed with $10 \%$ phosphoric acid $(2 \times 15 \mathrm{~mL})$ and brine $(20 \mathrm{~mL})$.

The organic layer was dried with $\mathrm{Na}_{2} \mathrm{SO}_{4}$ and evaporated under reduced pressure, and the crude oil was purified by flash chromatography (silicagel, eluant: 8:2 $n$-hexane/AcOEt with $1 \% \mathrm{HCOOH}$ ) to obtain pure 19 ( $408 \mathrm{mg}, 1.342 \mathrm{mmol}, 27 \%$ yield). 
Analytical characterization. ${ }^{1} \mathrm{H}-\mathrm{NMR}\left(\mathrm{CDCl}_{3}, 400 \mathrm{MHz}\right): \delta(\mathrm{ppm})=4.21-4.12(\mathrm{~m}, 2 \mathrm{H}), 2.38(\mathrm{t}$, $J=4.5 \mathrm{~Hz}, 2 \mathrm{H}), 2.32(\mathrm{t}, J=4.3 \mathrm{~Hz}, 2 \mathrm{H}), 1.73-1.59(\mathrm{~m}, 4 \mathrm{H}), 1.41-1.28(\mathrm{~m}, 8 \mathrm{H}), 1.01-0.97(\mathrm{~m}, 2 \mathrm{H}), 0.04(\mathrm{~s}$, 9H). ${ }^{13} \mathrm{C}-\mathrm{NMR}:\left(\mathrm{CDCl}_{3}, 100 \mathrm{MHz}\right): \delta(\mathrm{ppm})=177.93,173.60,62.58,34.32,34.22,29.46,29.48,29.30,29.10$, 25.12, 25.07, 17.05, -1.53 (3C). HR-ESI-MS: MW 325.1811 calcd. for $\mathrm{C}_{15} \mathrm{H}_{30} \mathrm{O}_{4} \mathrm{SiNa}$, MW 325.1815 found.

(1R,3aS,5aR,5bR,9S,11aR)-3a-(methoxycarbonyl)-5a,5b,8,8,11a-pentamethyl-1-(prop-1-en-2-yl)icosahydro-1H-cyclopenta[a]chrysen-9-yl 1-[2-(trimethylsilyl)ethyl] decanedioate/20. Dicyclohexylcarbodiimide (DCC, $201 \mathrm{mg}, 0.973 \mathrm{mmol}$ ) and dimethylaminopyridine (DMAP, $30 \mathrm{mg}, 0.243 \mathrm{mmol})$ were added under stirring to a solution of compound 18 (229 $\mathrm{mg}, 0.487 \mathrm{mmol})$ and compound $19(221 \mathrm{mg}, 0.731 \mathrm{mmol})$ in dry $\mathrm{CH}_{2} \mathrm{Cl}_{2}(5 \mathrm{~mL})$ at $0{ }^{\circ} \mathrm{C}$. The reaction was left stirring at $\mathrm{RT}$ overnight. Reaction monitoring (TLC, eluant: 9:1 n-hexane/AcOEt) confirmed the disappearance of starting material 18. The mixture was diluted with $\mathrm{CH}_{2} \mathrm{Cl}_{2}(10 \mathrm{~mL})$ and was filtered on a plug of celite. The solvent was removed under reduced pressure, and the resulting crude oil was purified by flash chromatography (silicagel, eluant: 96:4 n-hexane/AcOEt) to obtain pure 20 (339 mg, 0.449 mmol, $92 \%$ yield).

Analytical characterization. ${ }^{1} \mathrm{H}-\mathrm{NMR}\left(\mathrm{CDCl}_{3}, 400 \mathrm{MHz}\right): \delta(\mathrm{ppm})=4.74(\mathrm{bs}, 1 \mathrm{H}), 4.61(\mathrm{bs}, 1 \mathrm{H})$, $4.48(\mathrm{dd}, J=10.1,6.2 \mathrm{~Hz}, 1 \mathrm{H}), 4.21-4.12(\mathrm{~m}, 2 \mathrm{H}), 3.67(\mathrm{~s}, 3 \mathrm{H}), 3.06-2.95(\mathrm{~m}, 1 \mathrm{H}), 2.33-2.13(\mathrm{~m}, 6 \mathrm{H})$, 1.97-1.82 (m, 2H), $1.69(\mathrm{~s}, 3 \mathrm{H}), 1.47-1.34(\mathrm{~m}, 8 \mathrm{H}), 0.97(\mathrm{~s}, 3 \mathrm{H}), 0.92(\mathrm{~s}, 3 \mathrm{H}), 0.85(\mathrm{~s}, 3 \mathrm{H}), 0.84(\mathrm{~s}, 6 \mathrm{H})$, 0.05 (s, 9H). ${ }^{13} \mathrm{C}-\mathrm{NMR}:\left(\mathrm{CDCl}_{3}, 100 \mathrm{MHz}\right): \delta(\mathrm{ppm})=176.67,174.00,173.64,150.57,109.63,80.61,62.37$, $56.57,55.45$ (2C), 51.25, 50.46, 49.48, 47.01, 42.40, 40.70, 38.40, 38.27, 37.85, 37.12, 36.98, 34.82, 34.52, $34.27,32.18,30.61,29.68,29.10$ (3C), 27.97, 25.49, 25.12, 24.95, 23.75, 20.91, 19.36, 18.19, 17.33, 16.57, 16.18, 15.96, 14.69, -1.47 (3C). HR-ESI-MS: MW 777.5465 calcd. for $\mathrm{C}_{46} \mathrm{H}_{78} \mathrm{O}_{6} \mathrm{SiNa}$, MW 777.5469 found. Optical rotation, $[\alpha]_{D}^{20}:+10.2^{\circ}$.

10-\{[(1R,3aS,5aR,5bR,9S,11aR)-3a-(methoxycarbonyl)-5a,5b,8,8,11a-pentamethyl-1-(prop-1-en-2-yl) -icosahydro-1H-cyclopenta[a]chrysen-9-yl]oxy\}-10-oxodecanoic acid/21. Tetrabutylammonium fluoride (TBAF, $0.61 \mathrm{~mL}, 2.11 \mathrm{mmol}$ ) was added under stirring to a solution of compound 19 (318 $\mathrm{mg}$, $0.421 \mathrm{mmol})$ in dry THF (15 mL), and the reaction mixture was stirred at RT overnight. Reaction monitoring (TLC, eluant: 9:1 $n$-hexane/AcOEt with 1\% $\mathrm{HCOOH}$ ) confirmed the disappearance of starting material 20. The reaction was quenched by an addition of sat. $\mathrm{NH}_{4} \mathrm{Cl}(10 \mathrm{~mL})$. The aqueous phase was extracted with AcOEt $(2 \times 10 \mathrm{~mL})$, the collected organic phases were dried with $\mathrm{Na}_{2} \mathrm{SO}_{4}$ and evaporated under reduced pressure to obtain pure 21 (258 $\mathrm{mg}, 0.393 \mathrm{mmol}, 93 \%$ yield).

Analytical characterization. ${ }^{1} \mathrm{H}-\mathrm{NMR}\left(\mathrm{CDCl}_{3}, 400 \mathrm{MHz}\right): \delta(\mathrm{ppm})=4.72(\mathrm{bs}, 1 \mathrm{H}), 4.58(\mathrm{bs}, 1 \mathrm{H})$, $4.44(\mathrm{dd}, J=10.1,6.2 \mathrm{~Hz}, 1 \mathrm{H}), 3.65(\mathrm{~s}, 3 \mathrm{H}), 3.03-2.94(\mathrm{~m}, 1 \mathrm{H}), 2.23-2.16(\mathrm{~m}, 6 \mathrm{H}), 1.92-1.82(\mathrm{~m}, 2 \mathrm{H}), 1.67$ $(\mathrm{s}, 3 \mathrm{H}), 1.47-1.34(\mathrm{~m}, 8 \mathrm{H}), 0.95(\mathrm{~s}, 3 \mathrm{H}), 0.90(\mathrm{~s}, 3 \mathrm{H}), 0.84(\mathrm{~s}, 3 \mathrm{H}), 0.82(\mathrm{~s}, 6 \mathrm{H}) .{ }^{13} \mathrm{C}-\mathrm{NMR}\left(\mathrm{CDCl}_{3}, 100\right.$ $\mathrm{MHz}): \delta(\mathrm{ppm}) 180.32,177.30,174.33,151.11,110.29,81.31,57.20,56.08$ (2C), 51.89, 51.10, 50.12, 47.63, $43.04,41.34,39.04,38.90,38.47,37.76,37.60,35.43,34.92,34.70,32.81,31.25,30.32,29.68$ (3C), 28.61, $26.13,25.73,25.29,24.38,21.56,20.00,18.84,17.21,16.81,16.59,15.33$. HR-ESI-MS: MW 677.4757 calcd. for $\mathrm{C}_{41} \mathrm{H}_{66} \mathrm{O}_{6} \mathrm{Na}$, MW 677.4761 found. Optical rotation, $[\alpha]_{D}^{20}:+12.9^{\circ}$.

(1R,3aS,5aR,5bR,9S,11aR)-3a-(methoxycarbonyl)-5a,5b,8,8,11a-pentamethyl-1-(prop-1-en-2-yl)icosahydro-1H-cyclopenta[a]chrysen-9-yl-1-[(2R,3R,4S,5R,6R)-6-\{[(2R,3R,4S,5R,6R)-6-(hydroxy met-hyl)-3,4,5-tris[(trimethylsilyl)oxy]oxan-2-yl]oxy\}-3,4,5-tris[(trimethylsilyl)oxy]oxan-2-yl]methyl decanedioate/22-mono and (1R,3aS,5aR,5bR,9S,11aR)-3a-(methoxycarbonyl)-5a,5b,8,8,11a-pentamethyl1-(prop-1-en-2-yl)-icosahydro-1H-cyclopenta[a]chrysen-9-yl 1-[(2R,3R,4S,5R,6R)-6- $\{[(2 \mathrm{R}, 3 \mathrm{R}, 4 \mathrm{~S}, 5 \mathrm{R}, 6 \mathrm{R})$ 6- $\{[(10-\{[(1 R, 3 a S, 5 a R, 5 b R, 9 S, 11 a R)-3 a-(m e t h o x y c a r b o n y l)-5 a, 5 b, 8,8,11 a-p e n t a-m e t h y l-1-(p r o p-1-e n-2-$ yl)-icosahydro-1H-cyclopenta[a]chrysen-9-yl]oxy\}-10-oxodecanoyl)oxy]-methyl\}-3,4,5-tris [(trimethylsilyl)oxy]oxan-2-yl]oxy\}-3,4,5-tris[(trimethylsilyl)oxy]oxan-2-yl]methyl decanedioate/23-bis. $\mathrm{EDC} \cdot \mathrm{HCl}(15 \mathrm{mg}, 0.0763 \mathrm{mmol})$ and DMAP (1 mg, $0.00763 \mathrm{mmol})$ were added under stirring at RT to a solution of hexaTMS-protected trehalose 5 [40]. (59 $\mathrm{mg}, 0.0763 \mathrm{mmol})$ in anhydrous toluene (4 mL). After $30 \mathrm{~min}$, compound 21 (50 mg, $0.0763 \mathrm{mmol}$ ) was added, and the reaction mixture was stirred at $50{ }^{\circ} \mathrm{C}$ overnight. Reaction monitoring (TLC, eluant: 7:3 n-hexane/AcOEt) confirmed the disappearance of starting material 21. The solvent was then removed under reduced pressure, and the crude oil was 
purified by flash chromatography (silicagel, eluant: 85:15 $n$-hexane/AcOEt) to obtain pure 22-mono (23 mg, $0.0163 \mathrm{mmol}, 21 \%$ yield) and pure 23-bis (24 mg, $0.0117 \mathrm{mmol}, 15 \%$ yield).

Analytical characterization. 22-mono: ${ }^{1} \mathrm{H}-\mathrm{NMR}\left(\mathrm{CD}_{3} \mathrm{OD}, 400 \mathrm{MHz}\right): \delta(\mathrm{ppm})=4.96(\mathrm{dd}, \mathrm{J}=3.0$, $1.9 \mathrm{~Hz}, 2 \mathrm{H}), 4.74(\mathrm{~d}, J=2.1 \mathrm{~Hz}, 1 \mathrm{H}), 4.67-4.58(\mathrm{~m}, 1 \mathrm{H}), 4.47(\mathrm{dd}, J=10.6,5.7 \mathrm{~Hz}, 1 \mathrm{H}), 4.43-4.34(\mathrm{~m}$, $1 \mathrm{H}), 4.12-4.02(\mathrm{~m}, 2 \mathrm{H}), 3.99(\mathrm{td}, J=9.0,3.1 \mathrm{~Hz}, 2 \mathrm{H}), 3.87(\mathrm{dt}, J=9.5,3.0 \mathrm{~Hz}, 1 \mathrm{H}), 3.70-3.68(\mathrm{~m}, 5 \mathrm{H})$, 3.61-3.45 (m, 4H), 3.34-3.32 (m, 2H), $3.02(\mathrm{td}, J=10.8,4.7 \mathrm{~Hz}, 1 \mathrm{H}), 2.44-2.23(\mathrm{~m}, 6 \mathrm{H}), 1.90(\mathrm{tt}, J=11.7$, $5.8 \mathrm{~Hz}, 2 \mathrm{H}), 1.78-1.70(\mathrm{~m}, 6 \mathrm{H}), 1.36(\mathrm{~s}, 3 \mathrm{H}), 1.04(\mathrm{~s}, 3 \mathrm{H}), 0.97$ (s, 3H), $0.92(\mathrm{~s}, 3 \mathrm{H}), 0.89$ (s, 3H), 0.88 $(\mathrm{s}, 3 \mathrm{H}), 0.25-0.14(\mathrm{~m}, 54 \mathrm{H}) .{ }^{13} \mathrm{C}-\mathrm{NMR}\left(\mathrm{CD}_{3} \mathrm{OD}, 400 \mathrm{MHz}\right): \delta(\mathrm{ppm})=176.69,173.89,173.68,150.33$, $108.96,94.45,94.19,80.81,73.58,73.54,73.31,72.66$ (2C), 72.01, 71.21, 70.72, 62.91, 60.41, 56.48, 55.45 (2C), 50.44, 49.24, 47.09, 42.17, 40.53, 38.24, 38.19, 37.52, 36.91, 36.47, 34.16, 34.06, 33.62, 31.74, 30.24, $29.42,28.78$ (2C) $, 28.73,28.62,27.22,25.38,24.80,24.58,23.38,20.72,18.21,17.90,15.75,15.42,15.20$, 13.83, $(0.19,-0.25,-0.99,-1.07=18 \mathrm{C})$. HR-ESI-MS: MW 1433.8185 calcd. for $\mathrm{C}_{71} \mathrm{H}_{134} \mathrm{O}_{16} \mathrm{Si}_{6} \mathrm{Na}, \mathrm{MW}$ 1433.8191 found. Optical rotation, $[\alpha]_{D}^{20}:+60.3^{\circ}$. 23-bis: ${ }^{1} \mathrm{H}-\mathrm{NMR}\left(\mathrm{CDCl}_{3}, 400 \mathrm{MHz}\right.$, detected signals): $\delta(\mathrm{ppm})=4.94(\mathrm{~d}, J=3.0 \mathrm{~Hz}, 2 \mathrm{H}), 4.76(\mathrm{~d}, J=2.0 \mathrm{~Hz}, 2 \mathrm{H}), 4.62(\mathrm{~d}, J=3.2 \mathrm{~Hz}, 2 \mathrm{H}), 4.53-4.44(\mathrm{~m}, 2 \mathrm{H})$, $4.29(\mathrm{dd}, J=11.8,2.0 \mathrm{~Hz}, 2 \mathrm{H}), 4.07(\mathrm{dd}, J=11.8,4.3 \mathrm{~Hz}, 2 \mathrm{H}), 4.02(\mathrm{ddd}, J=9.4,4.2,2.1 \mathrm{~Hz}, 2 \mathrm{H}), 3.92$ $(\mathrm{t}, J=9.0 \mathrm{~Hz}, 2 \mathrm{H}), 3.69(\mathrm{~s}, 6 \mathrm{H}), 3.50(\mathrm{t}, J=9.0 \mathrm{~Hz}, 2 \mathrm{H}), 3.46(\mathrm{dd}, J=9.3,3.1 \mathrm{~Hz}, 2 \mathrm{H}), 3.01(\mathrm{td}, J=10.8$, $4.2 \mathrm{~Hz}, 2 \mathrm{H}), 2.40-2.16(\mathrm{~m}, 12 \mathrm{H}), 1.98-1.83(\mathrm{~m}, 4 \mathrm{H}), 1.71(\mathrm{~s}, 6 \mathrm{H}), 1.68-1.56(\mathrm{~m}, 16 \mathrm{H}), 1.28(\mathrm{~s}, 6 \mathrm{H}), 0.98(\mathrm{~s}$, $6 \mathrm{H}), 0.93(\mathrm{~s}, 6 \mathrm{H}), 0.86(\mathrm{~s}, 6 \mathrm{H}), 0.85(\mathrm{~s}, 12 \mathrm{H}), 0.16(\mathrm{~m}, 54 \mathrm{H})$.

${ }^{13} \mathrm{C}-\mathrm{NMR}\left(\mathrm{CDCl}_{3}, 100 \mathrm{MHz}\right): \delta(\mathrm{ppm})=176.66(2 \mathrm{C}), 173.68(2 \mathrm{C}), 173.60(2 \mathrm{C}), 150.55(2 \mathrm{C}), 109.62$ (2C), $94.44(2 \mathrm{C}), 80.61(2 \mathrm{C}), 73.49(2 \mathrm{C}), 72.67(2 \mathrm{C}), 71.94(2 \mathrm{C}), 70.75(2 \mathrm{C}), 63.31(2 \mathrm{C}), 56.57(2 \mathrm{C}), 55.46(2 \mathrm{C})$, $51.23(2 \mathrm{C}), 50.47(2 \mathrm{C}), 49.49(2 \mathrm{C}), 47.01(2 \mathrm{C}), 42.40(2 \mathrm{C}), 40.71(2 \mathrm{C}), 38.40(2 \mathrm{C}), 38.27(2 \mathrm{C}), 37.84(2 \mathrm{C})$, 37.13 (2C), 36.97 (2C), $34.81(2 \mathrm{C}), 34.28(2 \mathrm{C}), 34.11(2 \mathrm{C}), 32.18(2 \mathrm{C}), 30.61(2 \mathrm{C}), 29.68(2 \mathrm{C}), 29.14(2 \mathrm{C})$, $29.11(2 \mathrm{C}), 29.09$ (2C), 27.97 (2C), 25.49 (2C), 25.12 (2C), 24.75 (2C), $23.75(2 \mathrm{C}), 20.91(2 \mathrm{C}), 19.35(2 \mathrm{C})$, 18.19 (2C), 16.56 (2C), 16.16 (2C), 15.96 (2C), 14.69 (2C), 14.10 (2C), (1.06, 0.88, 0.18 =18C). HR-ESI-MS: MW 2070.2939 calcd. for $\mathrm{C}_{112} \mathrm{H}_{198} \mathrm{O}_{21} \mathrm{Si}_{6} \mathrm{Na}$, MW 2070.2949 found. Optical rotation, $[\alpha]_{D}^{20}:+41.2^{\circ}$.

(1R,3aS,5aR,5bR,9S,11aR)-3a-(methoxycarbonyl)-5a,5b,8,8,11a-pentamethyl-1-(prop-1-en-2-yl)icosahydro-1H-cyclopenta[a]chrysen-9-yl 1-[(2R,3S,4S,5R,6R)-3,4,5-trihydroxy-6- $\{[(2 R, 3 R, 4 S, 5 S, 6 R)-3,4$, 5-trihydroxy-6-(hydroxymethyl)oxan-2-yl]oxy\}oxan-2-yl]methyl decanedioate/3-Be-mono. Acetic acid $(18 \mu \mathrm{L}, 0.324 \mathrm{mmol})$ was added under stirring at RT to a solution of 22-mono (23 $\mathrm{mg}, 0.0162 \mathrm{mmol})$ in $\mathrm{MeOH}(1 \mathrm{~mL})$, and the reaction mixture was stirred at $40{ }^{\circ} \mathrm{C}$ for two days. Reaction monitoring (TLC, eluant: 98:2 $\left.\mathrm{CH}_{2} \mathrm{Cl}_{2} / \mathrm{MeOH}\right)$ confirmed the disappearance of starting 22-mono. The solvent was then removed under reduced pressure to obtain pure target 3-Be-mono (13 mg, $0.0133 \mathrm{mmol}, 82 \%$ yield).

Analytical characterization. ${ }^{1} \mathrm{H}-\mathrm{NMR}\left(\mathrm{CDCl}_{3}, 400 \mathrm{MHz}\right): \delta(\mathrm{ppm})=4.98(\mathrm{dd}, J=3.0,1.9 \mathrm{~Hz}, 2 \mathrm{H})$, $4.76(\mathrm{~d}, J=2.0 \mathrm{~Hz}, 1 \mathrm{H}), 4.65-4.59(\mathrm{~m}, 1 \mathrm{H}), 4.52-4.42(\mathrm{~m}, 1 \mathrm{H}), 4.45-4.37(\mathrm{~m}, 1 \mathrm{H}), 4.11-4.02(\mathrm{~m}, 2 \mathrm{H}), 3.97$ $(\mathrm{td}, J=8.9,3.1 \mathrm{~Hz}, 2 \mathrm{H}), 3.84(\mathrm{dt}, J=9.4,3.1 \mathrm{~Hz}, 1 \mathrm{H}), 3.72-3.67(\mathrm{~m}, 5 \mathrm{H}), 3.60-3.43(\mathrm{~m}, 4 \mathrm{H}), 3.35-3.33(\mathrm{~m}$, $2 \mathrm{H}), 3.01(\mathrm{td}, J=10.8,4.7 \mathrm{~Hz}, 1 \mathrm{H}), 2.43-2.17(\mathrm{~m}, 6 \mathrm{H}), 1.98-1.85(\mathrm{~m}, 2 \mathrm{H}), 1.75-1.68(\mathrm{~m}, 6 \mathrm{H}), 1.28(\mathrm{~s}, 3 \mathrm{H})$, $0.98(\mathrm{~s}, 3 \mathrm{H}), 0.94(\mathrm{~s}, 3 \mathrm{H}), 0.87(\mathrm{~s}, 3 \mathrm{H}), 0.85(\mathrm{~s}, 6 \mathrm{H}) .{ }^{13} \mathrm{C}-\mathrm{NMR}\left(\mathrm{CDCl}_{3}, 100 \mathrm{MHz}\right): \delta(\mathrm{ppm})=176.64,173.80$, $173.72,150.50,109.65,94.42,94.15,80.74,73.59,73.51,73.30,72.66,72.62,72.04,71.25,70.68,62.90,60.37$, $56.56,55.44,51.24,50.46,49.49,47.00,42.40,40.71,38.40,38.26,37.85,37.13,36.96,34.82,34.28,34.18$, $34.12,34.09,32.18,30.62,29.69,29.35,29.17,28.01,25.48,25.14,24.81,23.75,20.92,19.38,18.21,16.61$, 16.18, 15.96, 14.71. HR-ESI-MS: MW 1001.5813 calcd. for $\mathrm{C}_{53} \mathrm{H}_{86} \mathrm{O}_{16} \mathrm{Na}$, MW 1001.5819 found. Optical rotation, $[\alpha]_{D}^{20}:+70.6^{\circ}$.

Attempted synthesis of (1R,3aS,5aR,5bR,9S,11aR)-3a-(methoxycarbonyl)-5a,5b,8,8,11a-pentamethyl1-(prop-1-en-2-yl)-icosahydro-1H-cyclopenta[a]chrysen-9-yl 1-[(2R,3S,4S,5R,6R)-6-\{[(2R,3R,4S,5S,6R)6- $\{[(10-\{[(1 R, 3 a S, 5 a R, 5 b R, 9 S, 11 a R)-3 a-(m e t h o x y c a r b o n y l)-5 a, 5 b, 8,8,11 a-p e n t a m e t h y l-1-(p r o p-1-e n-2-y l)-$ icosahydro-1H-cyclopenta[a]chrysen-9-yl]oxy\}-10-oxodecanoyl)oxy]methyl\}-3,4,5-trihydroxyoxan2-yl]oxy\}-3,4,5-trihydroxyoxan-2-yl]methyl decanedioate/4-Be-bis. Acetic acid (13 $\mu \mathrm{L}, 0.234 \mathrm{mmol})$ was added under stirring at RT to a solution of 23-bis (24 mg, $0.0117 \mathrm{mmol})$ in $\mathrm{MeOH}(1 \mathrm{~mL})$, and the reaction mixture was stirred at $40{ }^{\circ} \mathrm{C}$ for four days. Reaction monitoring (TLC, eluant: 98:2 $\mathrm{CH}_{2} \mathrm{Cl}_{2} / \mathrm{MeOH}$ ) showed the formation of a series of uncharacterizable degradation products. 


\subsection{NA Assembly and Characterization}

Mono-Sq-NA1. In accordance with standard solvent evaporation protocols [41] the squalene-trehalose conjugate 1-mono $(4.0 \mathrm{mg})$ was first dissolved in THF (1 mL) in a vial while stirring at RT. The resulting solution was added dropwise to a round bottom flask containing MilliQ grade distilled water $(2 \mathrm{~mL})$ under magnetic stirring (500 rpm). The resulting suspension was stirred for $5 \mathrm{~min}$, then THF was thoroughly evaporated under reduced pressure, obtaining pure mono-Sq-NA1 as an opalescent suspension ( $2 \mathrm{~mL}, 2 \mathrm{mg} / \mathrm{mL}$ ).

Bis-Sq-NA2. In accordance with standard solvent evaporation protocols [41] the squalene-trehalose conjugate 2-bis $(4.0 \mathrm{mg})$ was first dissolved in THF $(1 \mathrm{~mL})$ in a vial while stirring at RT. The resulting solution was added dropwise to a round bottom flask containing MilliQ grade distilled water $(2 \mathrm{~mL})$ under magnetic stirring (500 rpm). The resulting suspension was stirred for $5 \mathrm{~min}$, then THF was thoroughly evaporated under reduced pressure, obtaining pure bis-Sq-NA2 as opalescent suspension (2 mL, $2 \mathrm{mg} / \mathrm{mL})$.

Mono-Be-NA3. In accordance with standard solvent evaporation protocols [41] the betulinic acid-trehalose conjugate 3-mono $(4.0 \mathrm{mg})$ was first dissolved in THF $(1 \mathrm{~mL})$ in a vial while stirring at RT. The resulting solution was added dropwise to a round bottom flask containing MilliQ grade distilled water $(2 \mathrm{~mL})$ under magnetic stirring $(500 \mathrm{rpm})$. The resulting suspension was stirred for $5 \mathrm{~min}$, then THF was thoroughly evaporated under reduced pressure, obtaining pure mono-Be-NA3 as opalescent suspension $(2 \mathrm{~mL}, 2 \mathrm{mg} / \mathrm{mL})$.

NA Characterization. NAs were characterized by dynamic light scattering (DLS), using a 90 Plus Particle Size Analyzer from Brookhaven Instrument Corporation (Holtsville, NY, USA) operating at $15 \mathrm{~mW}$ of a solid-state laser $(\lambda=661 \mathrm{~nm})$, using a 90-degree scattering angle. The $\zeta$-potential was determined at $25^{\circ} \mathrm{C}$ using a 90 Plus Particle Size Analyzer from Brookhaven Instrument Corporation (Holtsville, NY, USA) equipped with an AQ-809 electrode, operating at an applied voltage of $120 \mathrm{~V}$. Each sample was diluted to a concentration of $0.2 \mathrm{mg} / \mathrm{mL}$ and sonicated for $3 \mathrm{~min}$ before each experiment. Ten independent measurements of $60 \mathrm{~s}$ duration were performed for each sample. Hydrodynamic diameters were calculated using Mie theory, considering the absolute viscosity and refractive index values of the medium to be $0.890 \mathrm{cP}$ and 1.33 , respectively. The same aqueous samples at a concentration of $0.2 \mathrm{mg} / \mathrm{mL}$ were used for $\zeta$-potential measurement, without any change for the ionic strength (no addition of $\mathrm{KCl}$ ). The $\zeta$-potential was calculated from the electrophoretic mobility of nanoparticles, by using the Smoluchowski theory [42].

\subsection{Biology}

Cell cultures. HeLa cells (ATCC: CCL-2) were cultured in DMEM with 10\% FBS, 1\% penicillin/ streptomicin and $1 \%$ glutamine in a humidified atmosphere of $5 \% \mathrm{CO}_{2}$ at $37^{\circ} \mathrm{C}$ (all reagents from Euroclone). Cultures were treated with lipid-trehalose conjugates or NAs for 2 or $48 \mathrm{~h}$ at $37^{\circ} \mathrm{C}$ at the concentration indicated in the text.

Cytotoxicity assay. We performed the 3-(4,5-dimethylthiazol-2-yl)-2,5-diphenyltetrazolium bromide (MTT) assay to measure culture vitality. HeLa cells were cultured in a 96-well plate at a concentration of $5 \times 10^{3}$ cell $/ \mathrm{cm}^{2}$ and incubated at $37^{\circ} \mathrm{C}$ for $24 \mathrm{~h}$. MTT was added in cell medium at a final concentration of $0.25 \mathrm{mg} / \mathrm{mL}$. Incubation lasted $30 \mathrm{~min}$ at $37^{\circ} \mathrm{C}$. Then, the medium was removed and formazan precipitates were collected in $200 \mu \mathrm{L}$ of DMSO. The absorbance measured at $570 \mathrm{~nm}$ using a spectrophotometer reflects cell viability. Cell viability was expressed as fold over control condition set at $100 \%$.

Autophagy assay. We assessed autophagy by monitoring LC3 conversion by western-blotting as previously described [43]. Briefly, upon a wash in PBS, cells were solubilized in RIPA buffer (150 mM $\mathrm{NaCl}, 50 \mathrm{mM}$ HEPES, 0.5\% NP40, 1\% sodium-deoxycholate). After $1 \mathrm{~h}$ under mild agitation, the lysate was clarified by centrifugation for $20 \mathrm{~min}$ at 16,000 g. All experimental procedures were performed at $4{ }^{\circ} \mathrm{C}$. Protein concentrations were evaluated via Bradford assay (Bio-Rad, Segrate, Italy). For Western blotting experiments, an equal amount of proteins was diluted with $0.25 \% 5 \mathrm{X}$ Laemmli buffer, separated 
onto 10\% SDS-PAGE gels and transferred onto nitrocellulose membrane (Sigma-Aldrich Italy, Milan, Italy) at $80 \mathrm{~V}$ for $120 \mathrm{~min}$ at $4{ }^{\circ} \mathrm{C}$.

Primary antibodies (source in parentheses) included: Mouse anti-LC3, 1:500 (Enzo Life Sciences AG, Lausen, Switzerland), and mouse anti $\beta$-actin 1:1000 (Sigma Aldrich Italy, Milan, Italy) which were applied overnight in blocking buffer $(20 \mathrm{mM}$ Tris, $\mathrm{pH} 7.4,150 \mathrm{mM} \mathrm{NaCl}, 0.1 \%$ Tween 20, and $5 \%$ nonfat dry milk). Proteins were detected using the ECL prime detection system (GE Healthcare). Images were acquired with the imaging ChemiDoc Touch system (Bio Rad Laboratory Italy, Segrate, Italy), and the optical density of the specific bands was measured with ImageLab software (Bio Rad).

\subsection{Statistical Analysis}

All data are reported as mean \pm standard error of the mean (SEM). The entire data-set was logged into GraphPad Prism and analyzed via unpaired Student's T-test (two classes) or ANOVA followed by Tukey's posthoc test (more than two classes). Number of experiments (n) and level of significance (p) are indicated throughout the text.

\section{Results}

\subsection{Synthesis of Target 1-mono and 2-bis Squalene-Trehalose Conjugates}

In order to obtain either a mono-(target compound 1-Sq-mono, 1:1 squalene-trehalose conjugate) and a bis-squalenylated trehalose construct (target compound 2-Sq-bis, 2:1 squalene-trehalose conjugate), we focused our attention onto the hexaTMS-protected trehalose derivative 5 [38] and the carboxylated squalene-linker adduct 6 [31] (Figure 2).
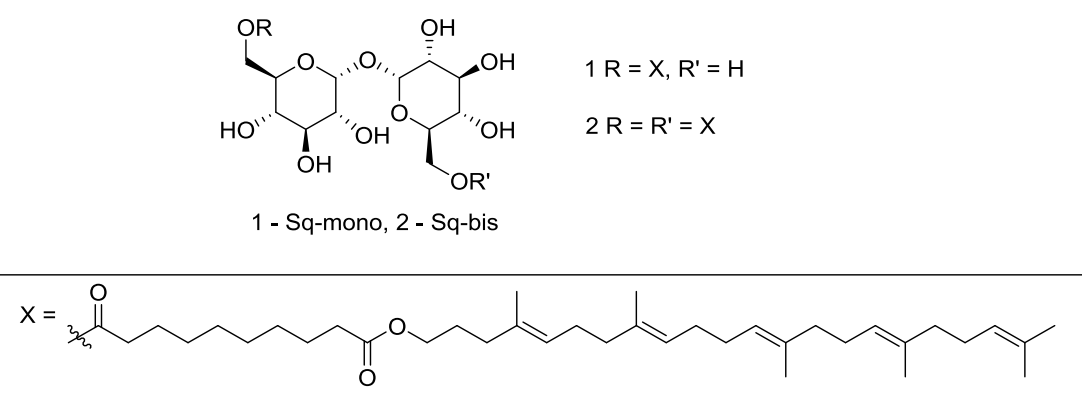

$\underbrace{\text { OTMS }}_{\text {OTMS }}$<smiles>CC(C)=CCC/C(C)=C/CC/C(C)=C/CC/C=C(\C)CC/C=C(\C)CCCOC(=O)CCCCCCCCCC(=O)O</smiles>

Figure 2. Chemical structure of target squalene-trehalose conjugates 1-Sq-mono and 2-Sq-bis, and of key synthetic intermediate hexaTMS-protected trehalose $\mathbf{5}$ and carboxylated squalene-linker adduct $\mathbf{6}$.

The synthesis of key intermediates 5 [38] and 6 [31] is reported respectively in Schemes 1 and 2.
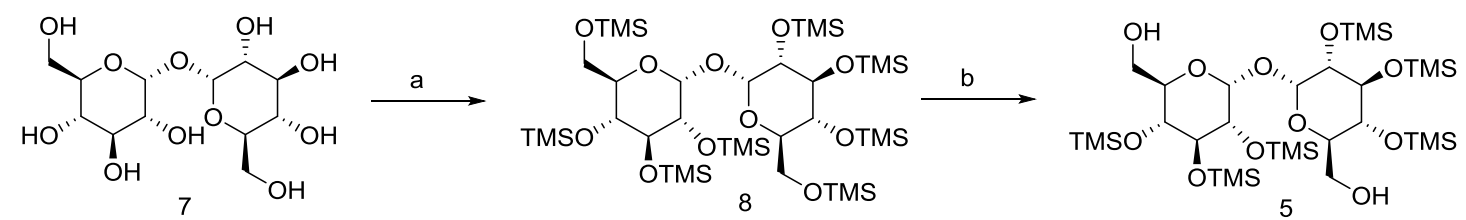

a: TMS-Cl, pyridine, $0^{\circ} \mathrm{C}$ to $\mathrm{rt}$, overnight; $\mathrm{b}: \mathrm{K}_{2} \mathrm{CO}_{3}, 3: 1 \mathrm{MeOH} / \mathrm{CH}_{2} \mathrm{Cl}_{2}, 0^{\circ} \mathrm{C}$ to $\mathrm{rt}, 90 \mathrm{~min}, 75 \%$ yield (2 steps)

Scheme 1. Synthesis of hexaTMS-protected trehalose 5. 


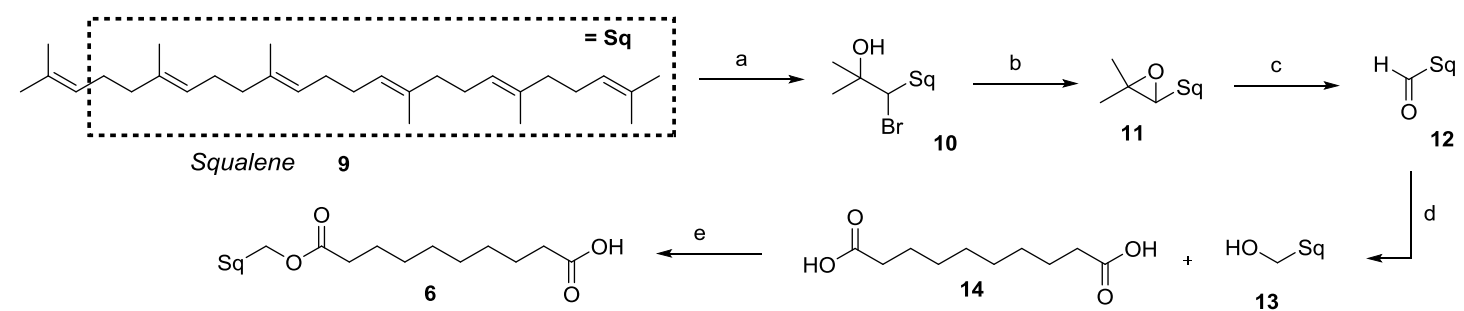

a: NBS, $\mathrm{H}_{2} \mathrm{O}$, THF, rt, 3h, 31\% yield; b: $\mathrm{K}_{2} \mathrm{CO}_{3}, \mathrm{MeOH}$, rt, $2 \mathrm{~h}$, quantitative yield; $\mathrm{c}: \mathrm{H}_{5} \mathrm{IO}$, dioxane, $\mathrm{H}_{2} \mathrm{O}, \mathrm{rt}, 2 \mathrm{~h}, \mathbf{8 6} \%$ yield; d: $\mathrm{NaBH}_{4}, \mathrm{MeOH}, \mathrm{rt}, 2 \mathrm{~h}, \mathbf{9 1} \%$ yield; e: $\mathrm{EDC} . \mathrm{HCl}, \mathrm{DMAP}, \mathrm{CH}_{2} \mathrm{Cl}_{2}$, rt, overnight, $\mathbf{6 0} \%$ yield.

Scheme 2. Synthesis of carboxylated squalene-linker adduct 6 .

HexaTMS-protected trehalose 5 was obtained through per-silylation of commercially-available trehalose $\mathbf{7}$ with TMS-Cl (per-silylated 8, step a), followed by a selective deprotection of primary, more easily accessible, hydroxyls (step b, Scheme 1).

Commercial squalene 9 was sequentially submitted to halohydration (bromohydrine 10, step a), base-promoted elimination (epoxide 11, step b), oxidative cleavage (aldehyde 12), reduction (alcohol 13) and mono-esterification with diacid 14, to provide target carboxylated squalene adduct 6 (step e, Scheme 2).

Finally, key intermediates 5 and $\mathbf{6}$ were coupled in equimolar amounts in an esterification protocol, obtaining a $\approx$ 3:1 mixture of 15-mono and 16-bis hexaTMS-protected compounds (step a, Scheme 3).
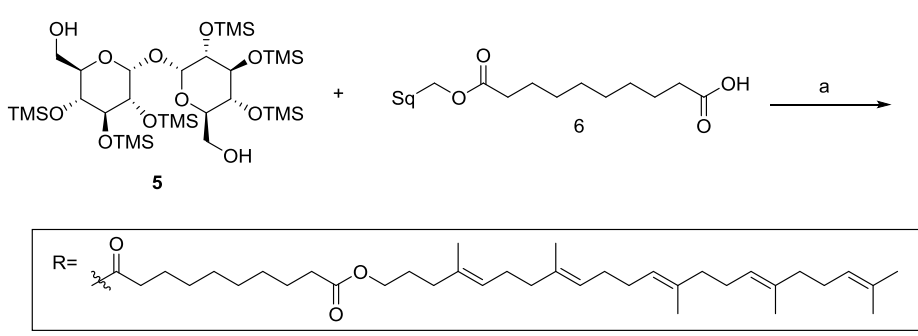

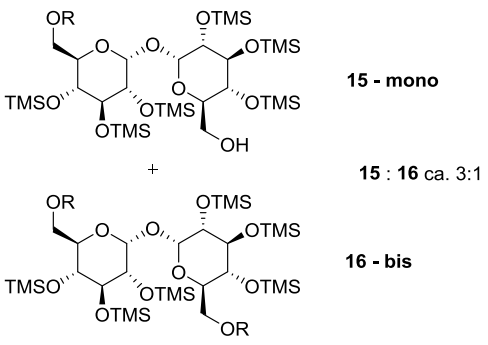

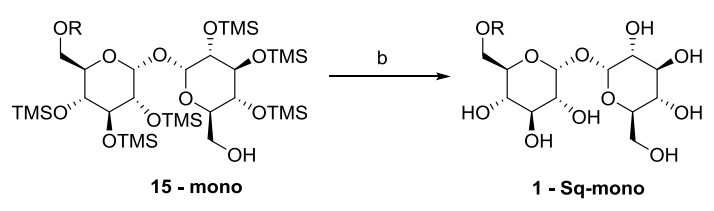
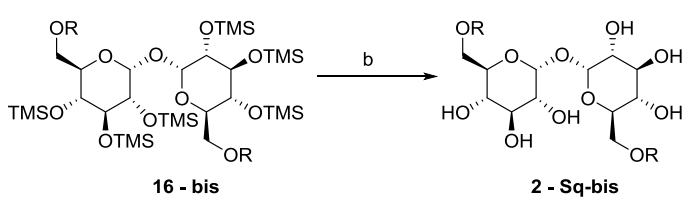

a: EDC. $\mathrm{HCl}$, DMAP, $\mathrm{CH}_{2} \mathrm{Cl}_{2}$, rt, overnight, $45 \%$ yield (15-mono), $14 \%$ yield ( 16 - bis):

b: AcOH, $\mathrm{MeOH}, 40^{\circ} \mathrm{C}$, overnight, quantitative yield (1 - Sq-mono), $90 \%$ yield (2 - Sq-bis).

Scheme 3. Synthesis of target 1-Sq-mono and 2-Sq-bis squalene-trehalose conjugates.

After chromatographic separation, both hexaTMS protected compounds 15-mono and 16-bis were submitted to acidic deprotection, yielding respectively 1-Sq-mono and 2-Sq-bis targets, respectively in $\mathbf{4 5 \%}$ and $\mathbf{1 3} \%$ overall yields from 5 and $\mathbf{6}$ (step b, Scheme 3 ).

3.2. Synthesis of Target 3-Be-mono Betulinic Acid-Trehalose Conjugate, Attempted Synthesis of Target 4-Be bis Betulinic Acid-Trehalose Conjugate

In order to obtain the target 3-Be-mono betulinic acid-trehalose conjugate we adopted a similar strategy, focusing our attention onto the same hexaTMS-protected trehalose derivative 5 [38] and the carboxylated betulinic-linker adduct 21 (Figure 3). 


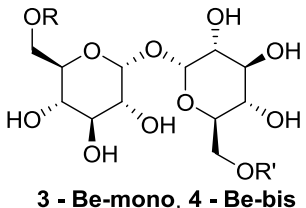

$3, \mathrm{R}=\mathrm{Be}, \mathrm{R}_{1}=\mathrm{H}$ $4, R=R_{1}=B e$

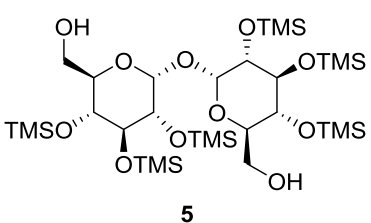

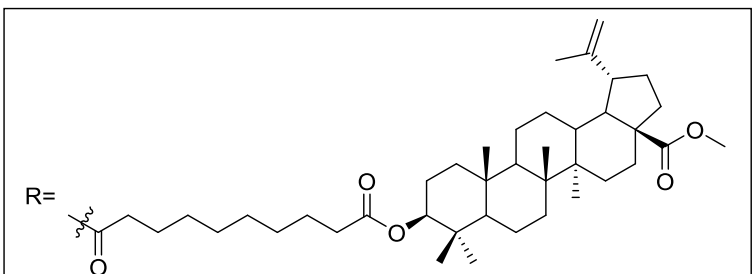

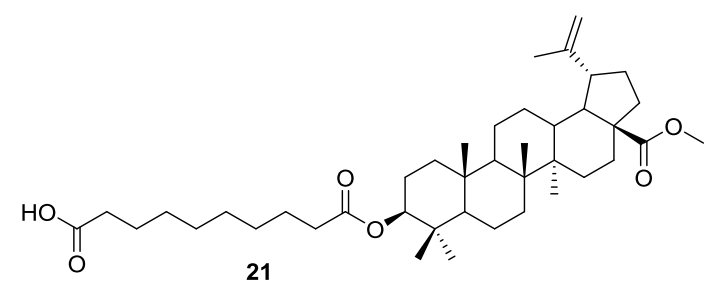

Figure 3. Chemical structure of target betulinic-trehalose conjugates 3-Be-mono and 4-Be-bis, of key synthetic intermediate hexaTMS-protected trehalose 5 and carboxylated betulinic-linker adduct 21.

The synthesis of key intermediate $\mathbf{2 1}$ is reported in Scheme 4.

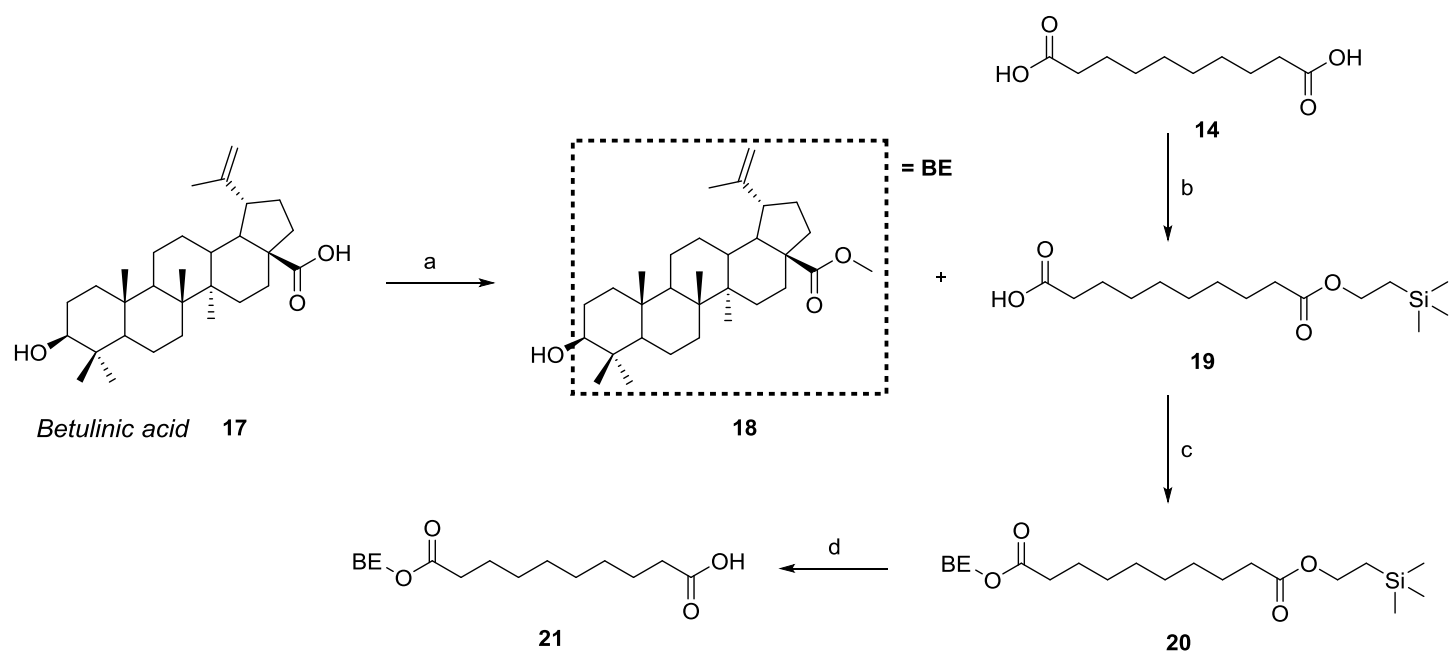

a: $\mathrm{Me}_{3} \mathrm{SiCH}=\mathrm{N}_{2}, \mathrm{MeOH}$, toluene, rt, overnight, $95 \%$ yield; b: trimethylsilyl ethanol, EDC $\mathrm{HCl}, \mathrm{DMAP}, \mathrm{CH}_{2} \mathrm{Cl}_{2}, \mathrm{Py}$, rt, overnight, $\mathbf{4 0} \%$ yield; $\mathrm{c}$ : DCC, DMAP, $\mathrm{CH}_{2} \mathrm{Cl}_{2}$, rt,overnight, $\mathbf{9 2} \%$ yield; $\mathrm{d}$ : TBAF, THF, rt, overnight, $\mathbf{9 3} \%$ yield.

Scheme 4. Synthesis of carboxylated betulinic-linker adduct 21.

Betulinic acid 17 was first esterified (methyl ester 18, step a), then coupled with mono-protected diacid 19 (prepared by a controlled esterification of sebacic acid 14, step b) to provide silyl-protected construct 20 (step c). Carboxylic acid deprotection finally provided target carboxylated betulinic-linker adduct 21 (step d, Scheme 4).

Finally, key intermediates 5 and $\mathbf{2 1}$ were coupled in equimolar amounts in an esterification protocol, obtaining a $\approx 1.5: 1$ mixture of 22-mono and 23-bis hexaTMS-protected compounds (step a, Scheme 5).

After chromatographic separation, hexaTMS protected compound 22-mono was submitted to acidic deprotection, yielding target 3-Be-mono betulinic acid-trehalose conjugate, in $21 \%$ overall yield from 5 and 21 (step b, Scheme 5). The same reaction, targeting 4-Be-bis from 23-bis, leads to uncharacterizable degradation products. 


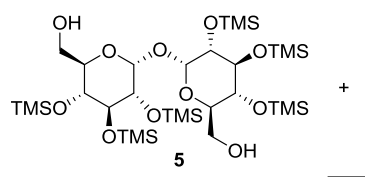
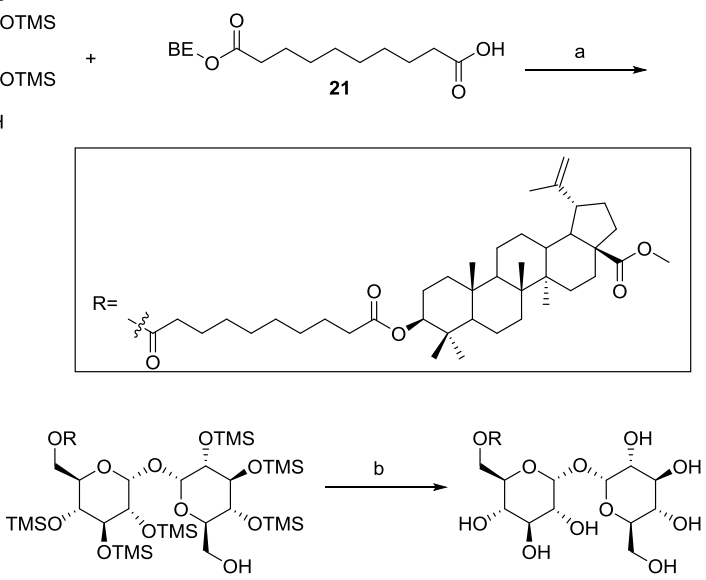

22

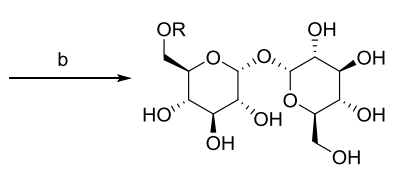

3 - Be-mono
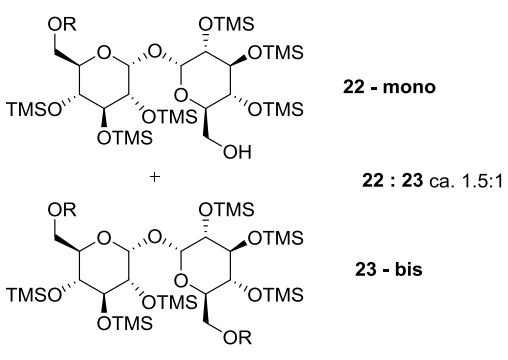

23 - bis

a: EDC $\mathrm{HCl}$, DMAP, toluene, $50^{\circ} \mathrm{C}$, overnight, $21 \%$ yield (22-mono), $15 \%$ yield $\left(23\right.$ - bis); b: $\mathrm{AcOH}, \mathrm{MeOH}, 40^{\circ} \mathrm{C}$, overnight, quantitative yield (3 - Be-mono), degradation (attempted synthesis of 4 - Be-bis).

Scheme 5. Synthesis of target 3-Be-mono betulinic-trehalose conjugate.

\subsection{NA Assembly and Structural Characterization}

Both 1-Sq-mono and 2-Sq-bis squalene-trehalose conjugates, and 3-Be-mono betulinic acid-trehalose conjugate were assembled into their corresponding NAs (mono-Sq-NA1, left, bis-Sq-NA2, middle, and mono-Be-NA3, right, Scheme 6) following a standard experimental protocol [38].
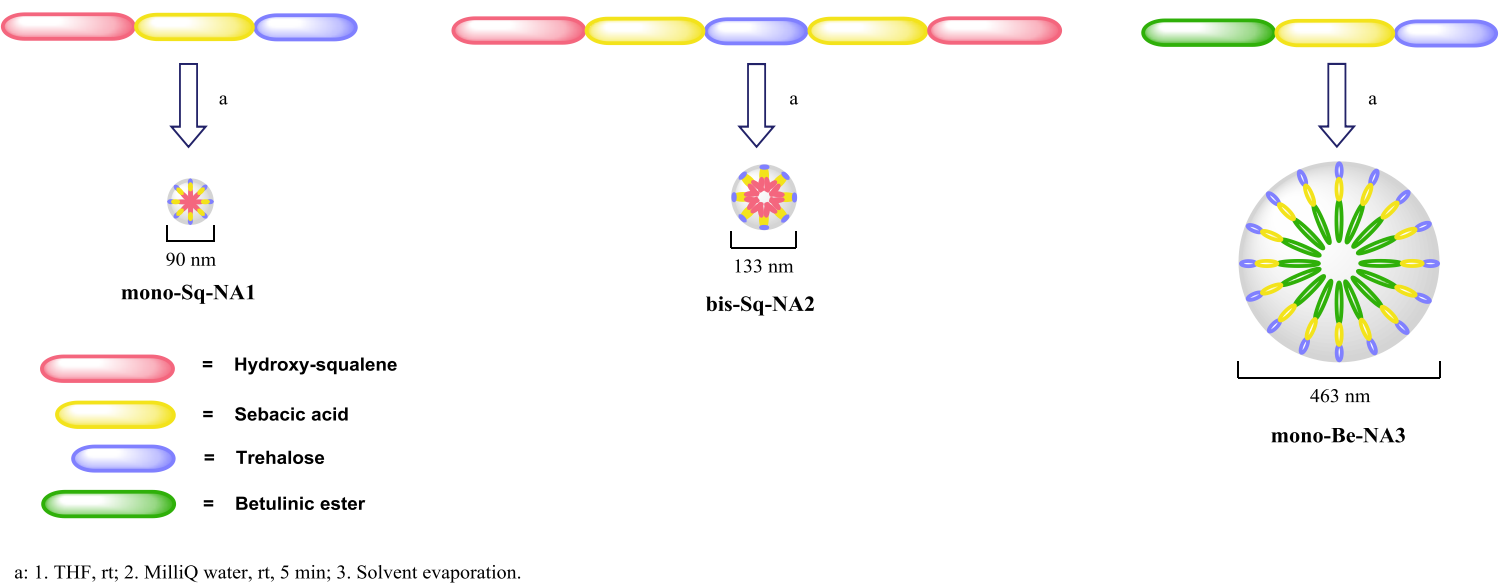

Scheme 6. Assembly and graphic representation of mono-Sq-NA1, bis-Sq-NA2 and mono-Be-NA3.

Self-assembled mono-Sq-NA1, bis-Sq-NA2 and mono-Be-NA3 were characterized in terms of hydrodynamic diameter and $\zeta$-potential, as shown in Table 1.

Table 1. Mono-Sq-NA1, mono-Be-NA3 and bis-Sq-NA2: characterization.

\begin{tabular}{cccc}
\hline Nanovector/Test & Hydrodynamic Diameter $(\mathrm{HD}, \mathbf{n m})$ & $\zeta$-Potential $(\mathbf{m V})$ & Polydispersity Index \\
\hline mono-Sq-NA1 & $90.4 \pm 0.7$ & $-25.12 \pm 0.79$ & $0.121 \pm 0.019$ \\
\hline bis-Sq-NA2 & $132.8 \pm 0.9$ & $-25.43 \pm 0.69$ & $0.072 \pm 0.010$ \\
\hline mono-Be-NA3 & $463 \pm 29$ & $-23.53 \pm 0.29$ & $0.126 \pm 0.010$ \\
\hline
\end{tabular}

The size of the hydrodynamic diameters shows an increase from mono-Sq-NA1 to bis-Sq-NA2, while the self-assembly of mono-Be-NA3 results in much larger NAs with a mean HD centered at 
about $460 \mathrm{~nm}$. However, the polydispersity index confirms the mono-dispersion of the colloidal solution of each NA. Moreover, the self-assembled NAs show good colloidal stability as confirmed by their $\zeta$-potential value $(<-20.0 \mathrm{mV})$, and by the stability of their hydrodynamic diameter (HD) which is not affected even after 10 days' storage in aqueous solution.

Furthermore, the TEM images and UV spectra of mono-Sq-NA1 (respectively Figures S1 and S2), of bis-Sq-NA2 (Figures S3 and S4) and of mono-Be-NA3 (Figures S5 and S6) are provided in the Supplementary Materials.

\subsection{Biological Profiling}

Finally, the set of three NAs was submitted to biological profiling in HeLa cells for cytotoxicity (safety determination) and autophagy induction (activity determination). Namely, we treated HeLa cultures for 2 and $48 \mathrm{~h}$ at $37^{\circ} \mathrm{C}$ with either the three NAs (either estimated, adjusted $20 \mu \mathrm{M}$ concentrations of trehalose in water for $2 \mathrm{~h}$, or estimated, adjusted $40 \mu \mathrm{M}$ concentrations for $48 \mathrm{~h}$ ), their non-assembled squalene-trehalose precursors 1-Sq-mono and 2-Sq-bis (either $20 \mu \mathrm{M}$ in DMSO for $2 \mathrm{~h}$, or $40 \mu \mathrm{M}$ for $48 \mathrm{~h}$ ) and betulinic acid-trehalose precursor 3-Be-mono (either $20 \mu \mathrm{M}$ in EtOH for $2 \mathrm{~h}$, or $40 \mu \mathrm{M}$ for $48 \mathrm{~h}$ ), or each individual component (100 mM trehalose in water, either $20 \mu \mathrm{M}$ squalene in DMSO and $20 \mu \mathrm{M}$ betulinic acid in EtOH for $2 \mathrm{~h}$, or $40 \mu \mathrm{M}$ with both for $48 \mathrm{~h}$ ), and relative vehicle (DMSO or $\mathrm{EtOH})$. Assays were carried out at $48 \mathrm{~h}$ to ensure the release of free trehalose from NAs.

At first, we determined the in vitro safety profile of each sample via the MTT cytotoxicity assay (Figure 4).
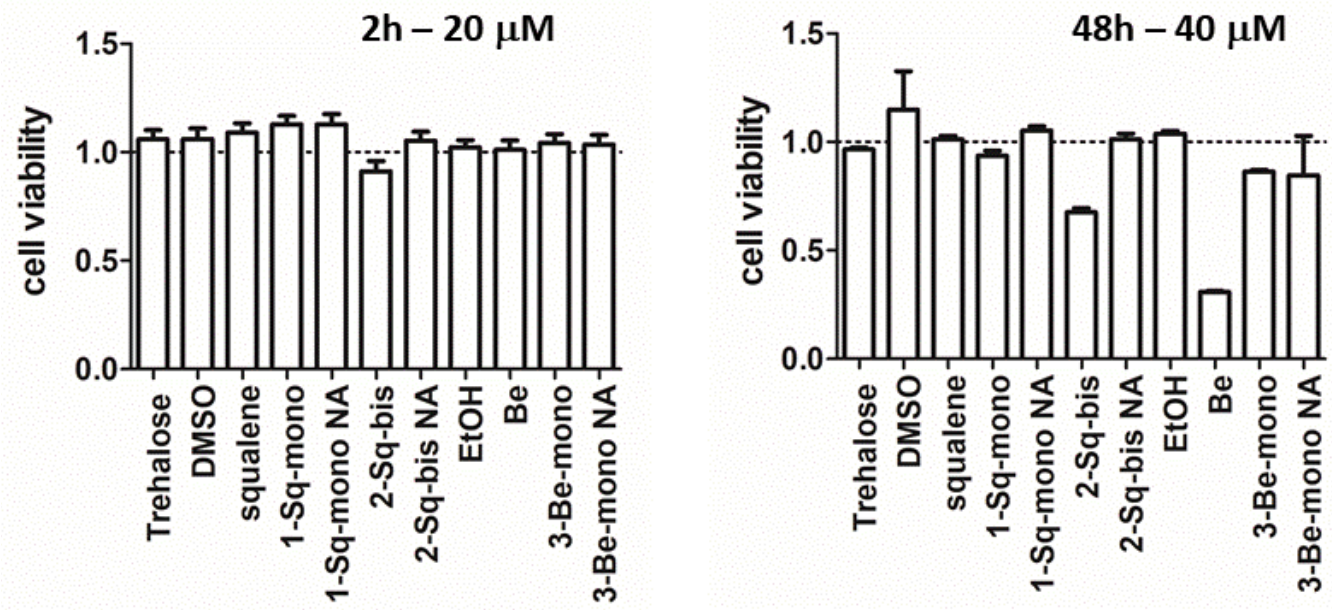

Figure 4. Cytotoxicity assay on betulinic acid, squalene, 1-Sq-mono, 2-Sq-bis, 3-Be-mono, mono-Sq-NA1, bis-Sq-NA2 and mono-Be-NA3. MTT assay, $2 \mathrm{~h} / 20 \mu \mathrm{M}$ (left) and $48 \mathrm{~h} / 40 \mu \mathrm{M}$ (right), trehalose $=100 \mathrm{mM}$.

The tested samples do not elicit an overt toxicity upon 2 hours of treatment. Instead, while the set of three NAs confirmed lack of cytotoxicity at $48 \mathrm{~h}$, both the 2 -Sq-bis construct $(\approx 65 \%$ viable cells at $48 \mathrm{~h})$ and free betulinic acid $(\approx 25 \%$ viable cells at $48 \mathrm{~h})$ show significant cytotoxicity $(p<0.001$ versus not treated, $n=4)$.

Next, we assessed if NAs, non-assembled precursors and individual components, could induce autophagy by western-blotting. In accordance with cytotox results, we tested NAs at both timelines ( $2 \mathrm{~h} /$ Figure 5, and $48 \mathrm{~h} /$ Figure 6), while non-assembled precursors and individual components were tested only at $2 \mathrm{~h}$. Autophagy can be monitored by tracking the mobility shift from LC3I to LC3II (Figures 5 and 6, right), that is a bona fide reporter of the induction of autophagy; and by the amount of LC3II, that correlates with the formation of autophagosomes (Figures 5 and 6, left). $\alpha$-Tubulin was used as an internal control in the assays. 

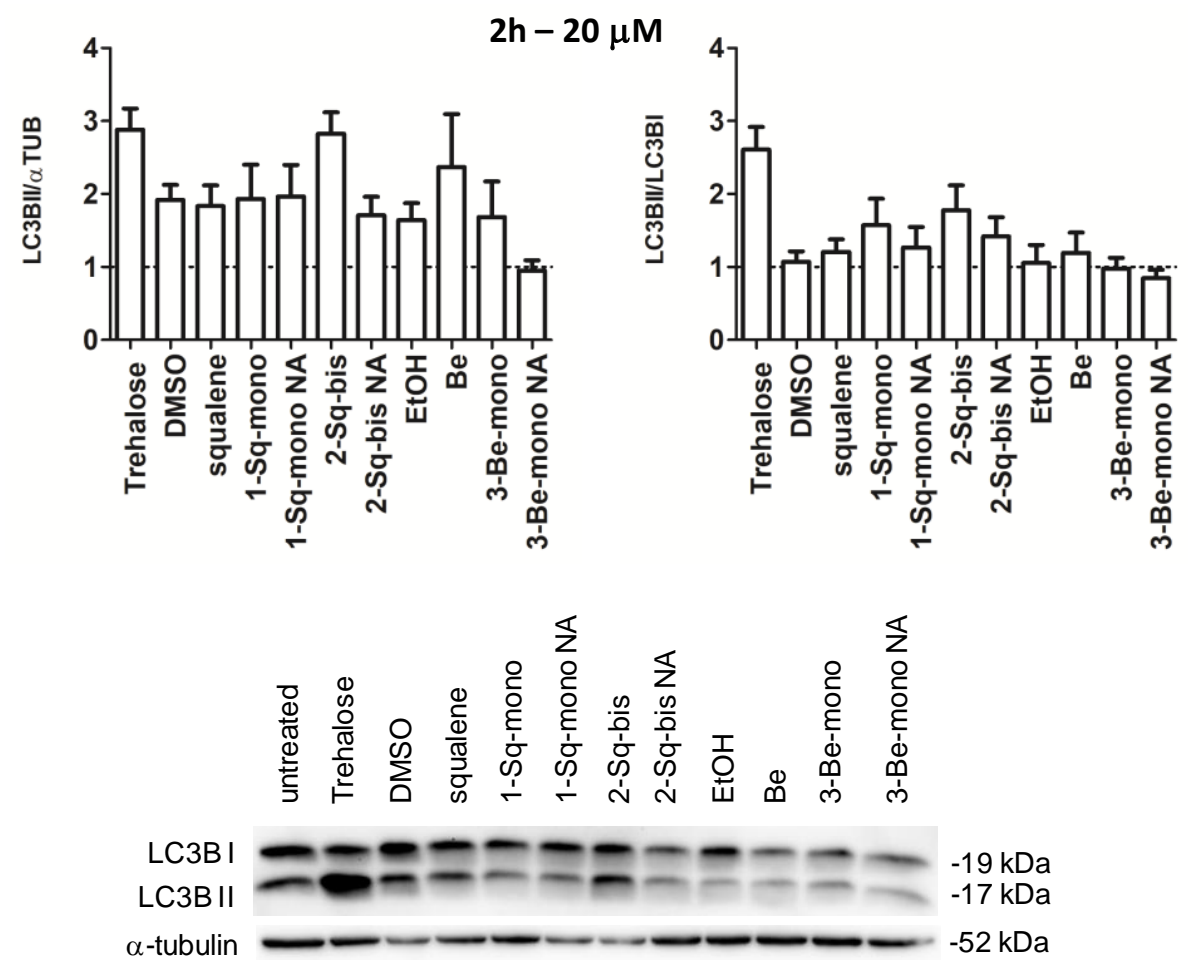

Figure 5. Autophagy induction on betulinic acid, squalene, 1-Sq-mono, 2-Sq-bis, 3-Be-mono, mono-Sq-NA1, bis-Sq-NA2 and mono-Be-NA3. LC3BII amount (left), LC3BII/LC3BI ratio (right), $2 \mathrm{~h} / 20 \mu \mathrm{M}$, trehalose $=100 \mathrm{mM})$.
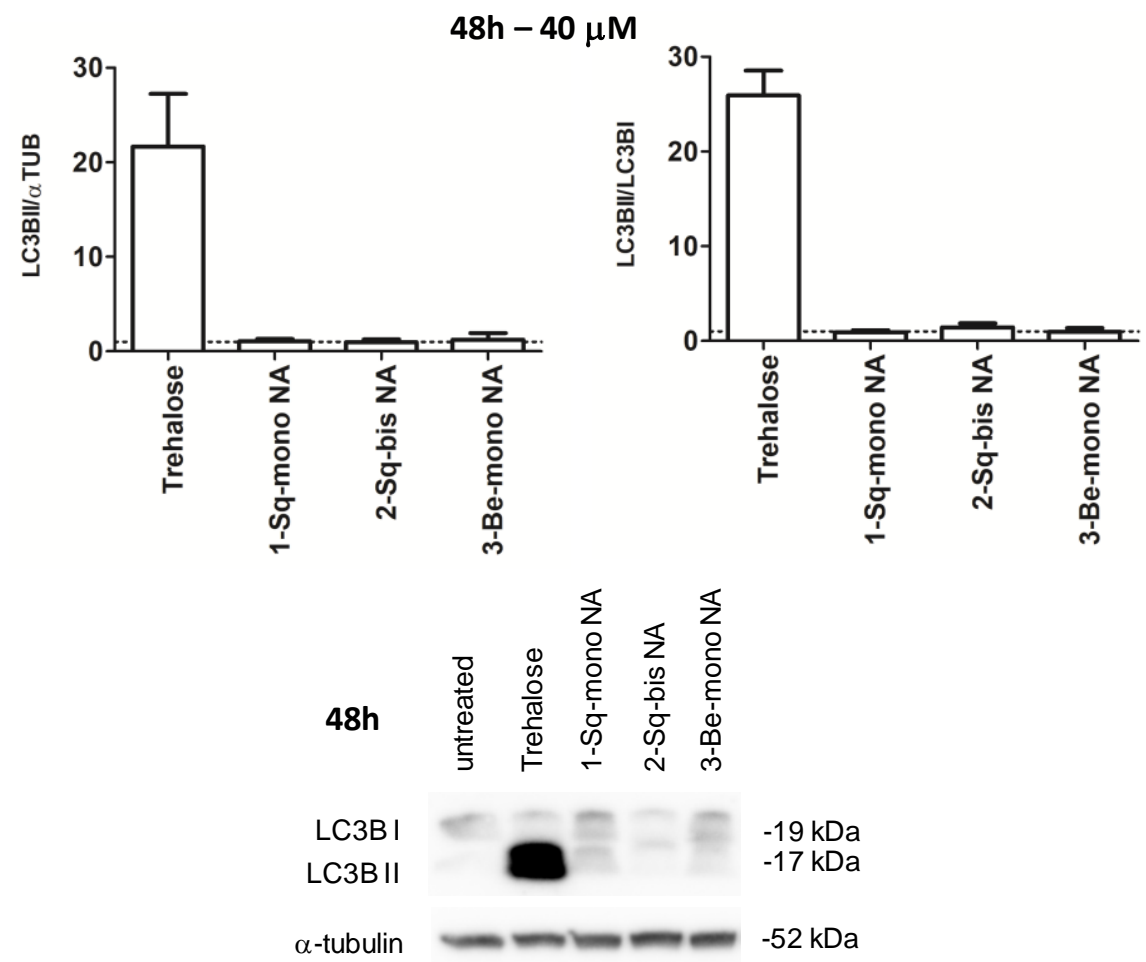

Figure 6. Autophagy induction on mono-Sq-NA1, bis-Sq-NA2 and mono-Be-NA3. LC3BII amount (left), LC3BII/LC3BI ratio (right), $48 \mathrm{~h} / 40 \mu \mathrm{M}$, trehalose $=100 \mathrm{mM}$ ).

At $2 \mathrm{~h}$, we only observed a moderate effect by the 2-Sq-bis construct that did not reach statistical significance (Figure 5, left and right). At $48 \mathrm{~h}$, surprisingly, the three NAs did not show any effect on autophagy induction/progress (Figure 6). 
We investigated the fate of our NAs in the biological medium, to rationalize their lack of biological effects. Thus, HeLa cell lysates were treated following a published procedure [44], obtaining two protein-free aqueous ( $\approx 4: 3 \mathrm{MeOH}$ :water) and organic $(\approx 3: 1 \mathrm{MeOH}$ :chloroform) layers. Their LC-MS analysis could neither detect trehalose as such at the expected $\mu \mathrm{M}$ concentration, nor the most likely lipid-trehalose intermediates (see Figures S7-S10, mono-Sq-NA1; and Figures S11-S14 mono-Be-NA3, Supplementary Materials). We could not rule out the presence of trehalose at lower, $\mathrm{nM}$ concentrations that would not elicit an autophagy-inducing effect in cells, due to its detection LC-MS limits; and we suggest that highly lipophilic lipid-trehalose intermediates 1-Sq-mono and 3-Be-mono remain trapped within the protein pellet.

\section{Discussion}

Accumulating evidence indicates that induction of autophagy can be clinically relevant in the context of neurodegenerative disorders characterized by protein aggregation [39]. Pre-clinically, trehalose alleviates protein aggregation and cellular toxicity in pathological deposition of amyloid/Alzheimer [22] and alpha synuclein/Parkinson [20]. It may act by binding to extra-cellular GLUT transporters and by inducing AMPK-dependent autophagy [19], and/or by cytosolic activation of the TFEP pathway [45]. Trehalose is highly hydrophilic, preventing its passive cell permeation [46]. Moreover, trehalases hydrolyze it to glucose at the GI barrier [23]. Thus, trehalose PK in humans is challenging. By conjugating trehalose with squalene and betulinic acid, and self-assembling three constructs into NAs, we generated entities with a putatively higher permeability profile, hopefully leading to higher effects on autophagy at lower dosages.

The 2:1 2-Sq-bis construct showed per se an indication of higher potency than trehalose on autophagy induction at much lower, $\approx 40 \mu \mathrm{M}$, concentrations. We hypothesize that its higher lipophilicity, compared to its 1:1 1-Sq-mono and 3-Be-mono counterparts, may yield better cell permeability after 2 hours of incubation, with a significant effect on autophagy induction.

The three NAs did not show any cytotoxicity, supporting their testing in the autophagy induction assay. The three mono-Sq-NA1, bis-Sq-NA2 and mono-Be-NA3 NAs did not show any effect upon autophagy induction; their inactivity could be justified by the absence of $\mu \mathrm{M}$-free trehalose in cell lysates (LC-MS determination), possibly due to limited degradation of NAs in $48 \mathrm{~h}$.

Our next efforts will include the design and execution of modified assays to measure autophagy induction at longer times and/or more NA degradation-prone conditions, and the synthesis of modified self-assembly trehalose-nanolipid conjugates to fine-tune their properties.

Supplementary Materials: The following are available online at http://www.mdpi.com/1999-4923/11/8/422/s1, Figure S1: TEM images of mono-Sq-NA1, Figure S2: UV-vis spectrum of mono-Sq-NA1, Figure S3: TEM images of bis-Sq-NA2, Figure S4: UV-vis spectrum of bis-Sq-NA2, Figure S5: TEM images of mono-Be-NA3, Figure S6: UV-vis spectrum of mono-Be-NA3, Figure S7: HPLC (220 nm, top) and TIC spectrum (ESI+, bottom) of the aqueous methanolic phase from the treatment of HeLa cells with mono-Sq-NA1/EC-37, Figure S8: MS analysis / compound searching for 2-Sq-bis (MW = 1448, first lane), 1-Sq-mono (MW=895, second lane), squalene alcohol $(\mathrm{MW}=386$, third lane) and trehalose (MW $=342 \mathrm{Da}$, fourth lane) in the aqueous methanolic phase from the treatment of HeLa cells with mono-Sq-NA1/EC-37, Figure S9: HPLC (220 nm, top) and TIC spectrum (ESI+, bottom) of the lipophilic methanol-chloroform phase from the treatment of HeLa cells with mono-Sq-NA1/EC-37, Figure S10: MS analysis / compound searching for 2-Sq-bis (MW = 1448, first lane), 1-Sq-mono (MW = 895, second lane), squalene alcohol ( $\mathrm{MW}=386$, third lane) and trehalose ( $\mathrm{MW}=342 \mathrm{Da}$, fourth lane) in the lipophilic methanol-chloroform phase from the treatment of HeLa cells with mono-Sq-NA1/EC-37, Figure S11: HPLC $(220 \mathrm{~nm}$, top) and TIC spectrum (ESI+, bottom) of the aqueous methanolic phase from the treatment of HeLa cells with mono-Be-NA3/MIC-17, Figure S12: MS analysis / compound searching for 3-Be-mono (MW = 979, first lane), betulinic acid ( $\mathrm{MW}=456$, second lane), betulinic acid methyl ester ( $\mathrm{MW}=470$, third lane) and trehalose (MW $=342$ $\mathrm{Da}$, fourth lane) in the aqueous methanolic phase from the treatment of HeLa cells with mono-Be-NA3/MIC-17, Figure S13: HPLC (220 nm, top) and TIC spectrum (ESI+, bottom) of the lipophilic methanol-chloroform phase from the treatment of HeLa cells with mono-Be-NA3/MIC-17, Figure S14: MS analysis / compound searching for 3-Be-mono ( $\mathrm{MW}=979$, first lane), betulinic acid ( $\mathrm{MW}=456$, second lane), betulinic acid methyl ester ( $\mathrm{MW}=470$, third lane) and trehalose ( $\mathrm{MW}=342 \mathrm{Da}$, fourth lane) in the lipophilic methanol-chloroform phase from the treatment of HeLa cells with mono-Be-NA3/MIC-17. 
Author Contributions: Conceptualization, P.S., G.P. and D.P.; chemistry, M.B. and E.C.; structural characterization, L.P.; biology, G.F.; bioanalytical studies, P.R.; writing—original draft preparation, E.C. and P.S.; HPLC-MS analysis, P.R.; structure elucidation, M.S.C.; writing-review and editing, P.S., G.P. and D.P.

Funding: This research was funded by Fondazione Telethon (grant number TDPG00514TA) and MIUR (PRIN-2017ENN4FY) for G.P.

Acknowledgments: D.P. expresses his gratitude to MAECI Italia-India Strategic Projects 2017-2019.

Conflicts of Interest: The authors declare no conflict of interest.

\section{References}

1. Farjadian, F.; Ghasemi, A.; Gohari, O.; Roointan, A.; Karimi, M.; Hamblin, M.R. Nanopharmaceuticals and nanomedicines currently on the market: Challenges and opportunities. Nanomedicine 2019, 14, 93-126. [CrossRef] [PubMed]

2. Ventola, C.L. Progress in nanomedicine: Approved and investigational nanodrugs. Pharm. Ther. 2017, 42, 742-755.

3. Bobo, D.; Robinson, K.J.; Islam, J.; Thurecht, K.J.; Corrie, S.R. Nanoparticle-based medicines: A review of FDA-approved materials and clinical trials to date. Pharm. Res. 2016, 33, 2373-2387. [CrossRef] [PubMed]

4. Jain, S.; Hirst, D.; O'Sullivan, J. Gold nanoparticles as novel agents for cancer therapy. Br. J. Radiol. 2012, 85, 101-113. [CrossRef] [PubMed]

5. Bharti, C.; Nagaich, U.; Pal, A.K.; Gulati, N. Mesoporous silica nanoparticles in target drug-delivery system: A review. Int. J. Pharm. Investig. 2015, 5, 124-133. [CrossRef] [PubMed]

6. Farjadian, F.; Moradi, S.; Hosseini, M. Thin chitosan films containing super-paramagnetic nanoparticles with contrasting capability in magnetic resonance imaging. J. Mater. Sci. Mater. Med. 2017, 28, 47. [CrossRef] [PubMed]

7. Maier-Hauff, K.; Ulrich, F.; Nestler, D.; Niehoff, H.; Wust, P.; Thiesen, B.; Orawa, H.; Budach, V.; Jordan, A. Efficacy and safety of intratumoral thermotherapy using magnetic iron-oxide nanoparticles combined with external beam radiotherapy on patients with recurrent glioblastoma multiforme. J. Neuro-Oncol. 2011, 103, 317-324. [CrossRef] [PubMed]

8. Schwenk, M.H. Ferumoxytol: A new intravenous iron preparation for the treatment of iron deficiency anemia in patients with chronic kidney disease. Pharmacotherapy 2010, 33, 70-79. [CrossRef] [PubMed]

9. Lasic, D.D. Doxorubicin in sterically stabilized liposomes. Nature 1996, 380, 561-562. [CrossRef] [PubMed]

10. Buster, J.E. Transdermal menopausal hormone therapy: Delivery through skin changes the rules. Expert Opin. Pharmacother. 2010, 11, 1489-1499. [CrossRef] [PubMed]

11. Turecek, P.L.; Bossard, M.J.; Schoetens, F.; Ivens, I.A. PEGylation of biopharmaceuticals: A review of chemistry and nonclinical safety information of approved drugs. J. Pharm. Sci. 2016, 105, 460-475. [CrossRef] [PubMed]

12. Awasthi, R.; Roseblade, A.; Hansbro, P.M.; Rathbone, M.J.; Dua, K.; Bebawy, M. Nanoparticles in cancer treatment: Opportunities and obstacles. Curr. Drug Targets 2018, 69, 1696-1709. [CrossRef] [PubMed]

13. Saeedi, M.; Eslamifar, M.; Khezri, K.; Dizaj, S.M. Applications of nanotechnology in drug delivery to the central nervous system. Biomed. Pharmacother. 2019, 111, 666-675. [CrossRef] [PubMed]

14. Hu, X.; Miller, L.; Richman, S.; Hitchman, S.; Glick, G.; Liu, S.; Zhu, Y.; Crossman, M.; Nestorov, I.; Gronke, R.S.; et al. A novel PEGylated interferon beta- 1a for multiple sclerosis: Safety, pharmacology, and biology. J. Clin. Pharmacol. 2012, 52, 798-808. [CrossRef] [PubMed]

15. Birch, G.G. Trehaloses. Adv. Carbohydr. Chem. 1963, 18, 201-225. [PubMed]

16. Richards, A.B.; Krakowka, S.; Dexter, L.B.; Schmid, H.; Wolterbeek, A.P.; Waalkens-Berendsen, D.H.; Shigoyuki, A.; Kurimoto, M. Trehalose: A review of properties, history of use and human tolerance, and results of multiple safety studies. Food Chem. Toxicol. 2003, 40, 871-898. [CrossRef]

17. Sussman, A.S.; Lingappa, B.T. Role of trehalose in ascaspores of Neurospora tetrasperma. Science 1959, 130, 1343. [CrossRef] [PubMed]

18. Van Dijck, P.; Colavizza, D.; Smet, P.; Thevelein, J.M. Differential importance of trehalose in stress resistance in fermenting and nonfermenting Saccharomyces cerevisiae cells. Appl. Environ. Microbiol. 1995, 61, 109-115. [PubMed]

19. Mardones, P.; Rubinsztein, D.C.; Hetz, C. Mystery solved: Trehalose kickstarts autophagy by blocking glucose transport. Sci. Signal. 2016, 9, fs2. [CrossRef] 
20. Tanaka, M.; Machida, Y.; Niu, S.; Ikeda, T.; Jana, N.R.; Doi, H.; Kurosawa, M.; Nekooki, M.; Nukina, N. Trehalose alleviates polyglutamine-mediated pathology in a mouse model of Huntington disease. Nat. Med. 2004, 10, 148-154. [CrossRef]

21. Jain, N.K.; Roy, I. Effect of trehalose on protein structure. Protein Sci. 2009, 18, 24-36. [CrossRef] [PubMed]

22. Liu, R.; Barkhordarian, H.; Emadi, S.; Park, C.B.; Sierks, M.R. Trehalose differentially inhibits aggregation and neurotoxicity of beta-amyloid 40 and 42. Neurobiol. Dis. 2005, 20, 74-81. [CrossRef] [PubMed]

23. Kalf, G.F.; Rieder, S.V. The purification and properties of trehalase. J. Biol. Chem. 1958, 230, 691-698. [PubMed]

24. Adhikari, P.; Pal, P.; Das, A.K.; Ray, S.; Bhattacharjee, A.; Mazumder, B. Nano lipid-drug conjugate: An integrated review. Int. J. Pharm. 2017, 529, 629-641. [CrossRef] [PubMed]

25. Couvreur, P.; Stella, B.; Reddy, L.H.; Mangenot, S.; Poupaert, J.H.; Desmaele, D.; Lepetre-Mouelhi, S.; Rocco, F.; Dereuddre-Bosquet, N.; Clayette, P.; et al. Squalenoyl nanomedicines as potential therapeutics. Nano Lett. 2006, 6, 2544-2548. [CrossRef] [PubMed]

26. Desmaele, D.; Gref, R.; Couvreur, P. Squalenoylation: A generic platform for nanoparticular drug delivery. J. Control. Release 2012, 161, 609-618. [CrossRef]

27. Semiramoth, N.; Di Meo, C.; Zouhiri, F.; Said-Hassane, F.; Valetti, S.; Gorges, R.; Nicolas, V.; Poupaert, J.H.; Chollet-Martin, S.; Desmaele, D.; et al. Selfassembled squalenoylated penicillin bioconjugates: An original approach for the treatment of intracellular infections. ACS Nano 2012, 6, 3820-3831. [CrossRef]

28. Buchy, E.; Valetti, S.; Mura, S.; Mougin, J.; Troufflard, C.; Couvreur, P.; Desmaële, D. Synthesis and cytotoxic activity of self-assembling squalene conjugates of 3-[(pyrrol-2-yl)methylidene]-2,3-dihydro-1H-indol-2-one anticancer agents. Eur. J. Org. Chem. 2015. [CrossRef]

29. Dash, S.K.; Giri, B. Self-assembled betulinic acid: A better alternative form of betulinic acid for anticancer therapy. J. Exp. Med. Biol. 2019, 1, 1-2.

30. Dash, S.K.; Dash, S.S.; Chattopadhyay, S.; Ghosh, T.; Tripathy, S.; Mahapatra, S.K.; Bag, B.G.; Das, D.; Roy, S. Folate decorated delivery of self-assembled betulinic acid nano fibers: A biocompatible antileukemic therapy. RCS Adv. 2015, 5, 24144. [CrossRef]

31. Fumagalli, G.; Marucci, C.; Christodoulou, M.S.; Stella, B.; Dosio, F.; Passarella, D. Self-assembly drug conjugates for anticancer treatment. Drug Discov. Today 2016, 21, 1321-1329. [CrossRef] [PubMed]

32. Borrelli, S.; Christodoulou, M.S.; Ficarra, I.; Silvani, A.; Cappelletti, G.; Cartelli, D.; Damia, G.; Ricci, F.; Zucchetti, M.; Dosio, F.; et al. New class of squalene-based releasable nanoassemblies of paclitaxel, podophyllotoxin, camptothecin and epothilone A. Eur. J. Med. Chem. 2014, 85, 179-190. [CrossRef] [PubMed]

33. Borrelli, S.; Cartelli, D.; Secundo, F.; Fumagalli, G.; Christodoulou, M.S.; Borroni, A.; Perdicchia, D.; Dosio, F.; Milla, P.; Cappelletti, G.; et al. Self-Assembled Squalene-based Fluorescent Heteronanoparticles. ChemPlusChem 2015, 80, 47-49. [CrossRef]

34. Fumagalli, G.; Mazza, D.; Christodoulou, M.S.; Damia, G.; Ricci, F.; Perdicchia, D.; Stella, B.; Dosio, F.; Sotiropoulou, P.A.; Passarella, D. Cyclopamine-paclitaxel-containing nanoparticles: Internalization in cells detected by confocal and super-resolution microscopy. ChemPlusChem 2015, 80, 1380-1383. [CrossRef]

35. Fumagalli, G.; Stella, B.; Pastushenko, I.; Ricci, F.; Christodoulou, M.S.; Damia, G.; Mazza, D.; Arpicco, S.; Giannini, C.; Morosi, L.; et al. Heteronanoparticles by self-assembly of doxorubicin and cyclopamine conjugates. ACS Med. Chem. Lett. 2017, 8, 953-957. [CrossRef] [PubMed]

36. Fumagalli, G.; Giorgi, G.; Vágvölgyi, M.; Colombo, E.; Christodoulou, M.S.; Collico, V.; Prosperi, D.; Dosio, F.; Hunyadi, A.; Montopoli, M.; et al. Hetero-nanoparticles by self-assembly of ecdysteroid and doxorubicin conjugates to overcome cancer resistance. ACS Med. Chem. Lett. 2018, 9, 468-471. [CrossRef] [PubMed]

37. Fumagalli, G.; Christodoulou, M.S.; Riva, B.; Revuelta, I.; Marucci, C.; Collico, V.; Prosperi, D.; Riva, S.; Perdicchia, D.; Bassanini, I.; et al. Self-assembled 4-(1,2-diphenylbut-1-en-1-yl)aniline based nanoparticles: Podophyllotoxin and aloin as building blocks. Org. Biomol. Chem. 2017, 15, 1106-1109. [CrossRef] [PubMed]

38. Fumagalli, G.; Polito, L.; Colombo, E.; Foschi, F.; Christodoulou, M.S.; Galeotti, F.; Perdicchia, D.; Bassanini, I.; Riva, S.; Seneci, P.; et al. Self-assembling releasable thiocolchicine-diphenylbutenylaniline conjugates. ACS Med. Chem. Lett. 2019, 10, 611-614. [CrossRef]

39. Guo, F.; Liu, X.; Cai, H.; Le, W. Autophagy in neurodegenerative diseases: Pathogenesis and therapy. Brain Pathol. 2018, 28, 3-13. [CrossRef] [PubMed]

40. Sarpe, A.V.; Kulkarni, S.S. Synthesis of maradolipid. J. Org. Chem. 2011, 76, 6866-6870. [CrossRef] [PubMed] 
41. Battaglia, L.; Gallarate, M. Lipid nanoparticles: State of the art, new preparation methods and challenges in drug delivery. Expert Opin. Drug Deliv. 2012, 9, 497-508. [CrossRef] [PubMed]

42. Von Smoluchowski, M. Contribution à la théorie de l'endosmose électrique et de quelques phenomènes. Pisma Mariana Smoluchowskiego 1924, 1, 403.

43. Klionski, D.J.; Abdelmohsen, K.; Abe, A.; Abedin, M.J.; Abeliovich, H.; Acevedo Arozena, A.; Adachi, H.; Adams, C.M.; Adams, P.D.; Adeli, K.; et al. Guidelines for the use and interpretation of assays for monitoring autophagy (3rd edition). Autophagy 2016, 12, 1-222. [CrossRef] [PubMed]

44. Fic, E.; Kedracka-Krok, S.; Jankowska, U.; Pirog, A.; Dziedzicka-Wasylewska, M. Comparison of protein precipitation methods for various rat brain structures prior of proteomic analysis. Electrophoresis 2010, 31, 3573-3579. [CrossRef] [PubMed]

45. Emanuele, E. Can trehalose prevent neurodegeneration? Insights from experimental studies. Curr. Drug Targets 2014, 15, 551-557. [CrossRef] [PubMed]

46. Rusmini, P.; Cortese, K.; Crippa, V.; Cristofani, R.; Cicardi, M.E.; Ferrari, V.; Vezzoli, G.; Tedesco, B.; Meroni, M.; Messi, E.; et al. Trehalose induces autophagy via lysosomal-mediated TFEB activation in models of motoneuron degeneration. Autophagy 2019, 15, 631-651. [CrossRef] [PubMed]

(C) 2019 by the authors. Licensee MDPI, Basel, Switzerland. This article is an open access article distributed under the terms and conditions of the Creative Commons Attribution (CC BY) license (http://creativecommons.org/licenses/by/4.0/). 